\title{
Moving contact lines and dynamic contact angles: a 'litmus test' for mathematical models, accomplishments and new challenges
}

\author{
Yulii D. Shikhmurzaev ${ }^{\mathrm{a}}$ \\ School of Mathematics, University of Birmingham, Birmingham B15 2TT, UK
}

Received 27 October 2019 / Accepted 6 July 2020

Published online 14 September 2020

\begin{abstract}
After a brief overview of the 'moving contact-line problem' as it emerged and evolved as a research topic, a 'litmus test' allowing one to assess adequacy of the mathematical models proposed as solutions to the problem is described. Its essence is in comparing the contact angle, an element inherent in every model, with what follows from a qualitative analysis of some simple flows. It is shown that, contrary to a widely held view, the dynamic contact angle is not a function of the contact-line speed as for different spontaneous spreading flows one has different paths in the contact angle-versus-speed plane. In particular, the dynamic contact angle can decrease as the contact-line speed increases. This completely undermines the search for the 'right' velocity-dependence of the dynamic contact angle, actual or apparent, as a direction of research. With a reference to an earlier publication, it is shown that, to date, the only mathematical model passing the 'litmus test' is the model of dynamic wetting as an interface formation process. The model, which was originated back in 1993, inscribes dynamic wetting into the general physical context as a particular case in a wide class of flows, which also includes coalescence, capillary breakup, freesurface cusping and some other flows, all sharing the same underlying physics. New challenges in the field of dynamic wetting are discussed.
\end{abstract}

\section{Introduction}

Capillary flows with moving contact lines have long become a research field in its own right studied in hundreds of research papers, periodically reviewed [1-7], summarized in bulky monographs [8-10] and occasionally erupting in a lively debate [11-20]. At the same time, over the years, the field accumulated its own collection of truisms, statements which in papers and presentations are often preceded with the words 'as we know'. On inspection, much of this accepted wisdom turns out to be simply old beliefs that guided early research in the field and then ossified as they became embedded in theories and interpretations of experiments. The inherent multidisciplinarity of dynamic wetting as a research area brings in researchers with different

a e-mail: Y.D.Shikhmurzaev@bham.ac.uk 
backgrounds many of whom come up with what they regard as 'mathematical models'. These models eagerly embrace the accepted views as something 'we know' and add refreshing new elements. They hybridize concepts taken from different modelling frameworks, use molecular scales in continuum models, add intermolecular forces on top of the continuum description, associate slip featuring in continuum models with molecular slip reported in molecular dynamics simulations, to mention but a few creative innovations. As a result of all these modelling practices analyzed, in particular, in [7], a newcomer in the field faces a formidable task of sifting through numerous publications, weeding out misconceptions and putting together a coherent view of the subject free from the dogmas taken on authority and unsubstantiated beliefs, seductive but misleading.

The scope of the present work is to help the newcomer by analyzing the mathematical modelling of dynamic wetting through the prism of some qualitative features of this type of flow that become apparent once the spontaneous spreading of liquids on solids is considered. The structure of the paper is as follows.

Section 2 gives a brief overview of dynamic wetting from the mathematical modelling viewpoint, outlines the prevailing ideas and how they converge on what is, mathematically, one and the same approach.

In Section 3, we describe a simple qualitative 'litmus test' which allows one to see immediately whether or not this or that mathematical model deserves further consideration. The test focusses on the key element of every theory formulated in the framework of continuum mechanics, namely the behaviour of the dynamic contact angle, and points out a class of capillary flows to be looked at. These flows show on a qualitative level whether or not this key element is correct in principle, on the level of the model's mathematical structure. In particular, the test allows one to conclude that the entire research direction aimed at derivation of an appropriate formula describing the dynamic contact angle, actual or apparent, as a function of the contact-line speed is a dead end: such a function does not exist in principle.

In Section 4, we summarize what is actually known on a theoretical level and give an example of a mathematical model which passes the 'litmus test' and describes all main features of dynamic wetting of a smooth chemically homogeneous non-reactive non-deformable solid substrate. The mathematical essence of this model is given in the Appendix.

Section 5 outlines some new challenges for theoretical research where experimentalists have already made substantial progress which is now awaiting its theoretical understanding.

Finally, Section 6 concludes the paper with some remarks regarding existing approaches to continuum mechanics as a branch of applied mathematics and as a modelling framework. It is argued that it is this duality of modern continuum mechanics not reflected in how this subject is being taught that is largely behind the misdirection of research effort in the area of dynamic wetting which this paper describes.

\section{An overview}

\subsection{The original 'moving contact-line problem'}

Dynamic wetting, i.e., the process of spreading of a liquid over a solid surface, has become the subject of experimental research more than a century ago $[21,22]$ and back then it was even part of simple engineering-level mathematical modelling aimed at describing the meniscus-driven flow in a capillary tube [23,24]. However, it was put on the agenda of the theoretical fluid mechanics community as a topic for research 
only in 1971 by a seminal paper of Huh and Scriven [25]. Huh and Scriven considered a somewhat artificial situation where an interface between two immiscible incompressible viscous fluids, instead of being subject to the normal-stress boundary condition, is planar and the contact line it forms with a flat solid surface moves across the latter. Then, in the reference frame moving with the contact line, in each of the fluids occupying a wedge-shaped domain the flow is driven by the solid boundary moving parallel to itself due to the no-slip boundary condition imposed on it, whilst on the interface between the fluids the flow is subject to the impermeability condition together with the continuity of the tangential velocity and stress. This was a generalization of an earlier work by Moffatt [26], known now primarily for discovering Moffatt's eddies, where the flow of one viscous incompressible fluid in a wedge region subject to the no-slip condition on the solid boundary and zero normal velocity and tangential stress on the free surface was considered.

Despite the promising title of Huh and Scriven's paper ('Hydrodynamic model of steady movement of a solid/liquid/fluid contact line'), it did not propose any model nor did it offer any solution; it posed a problem. The authors showed that, in their simplified formulation, the shear stress exerted by the fluids on the solid boundary is nonintegrably singular so that, according to the classical model they examined, viscous fluids simply could not spread on solids. This outcome obviously contradicts the physical reality we observe on a daily basis and "signals failure of one or more hypotheses underlying the model" [25]. Thus, the 'moving contact-line problem' was born as 'the problem of a non-integrable shear-stress singularity'.

However, the original moving contact-line problem was short-lived. Very soon it has been shown [27] that it is the no-slip condition that is responsible for the singularity and that any slip condition used instead makes shear stress integrable [28]. Furthermore, as it turned out, the form of the slip condition has an asymptotically vanishing effect on the flow in the far field, i.e., on the scale large compared with the size of the region near the contact line where no slip is replaced by slip [28], whilst the far-field flow is that described by Huh and Scriven.

This happy outcome provided a fertile ground for many theoretical exercises [29-36] all proposing, again and again, the 'right' slip conditions and then applying the result to model problems. Sometimes, a slip condition is not even formulated in its mathematical form, and the proposed model is numerical from the start, with the singularity removed via mesh-dependent 'numerical slip' implicitly built into the discretization of the problem [37,38]. Mentioned here only for completeness of our overview, this numerics-solves-all approach is obviously on the wrong side of the elusive divide between pragmatism and incompetence in the use of numerical methods, as pointed out, e.g., in [39].

The most popular choice of the slip condition to date remains the Navier condition [40] and its numerous generalizations; see, e.g., Sect. 9 of [41] or Chap. 3 of [10] for a review.

Although the slip versus no-slip issue was settled back in the 1970s and in serious research reappears only as a rhetorical 'slip or no slip?' question [42], one can occasionally come across works claiming to have resolved the problem with the no-slip condition intact but invariably only in the lubrication approximation, e.g., [43]. This apparent paradox has a simple explanation. If one considers the liquid spreading flow for small slopes, with the free surface subject to the normal-stress boundary condition, using the lubrication approximation, then the singularity will not disappear but, thanks to the approximation, it will have a simpler form and manifest itself in the impossibility of the free surface to touch the solid. Then, the singularity can be suppressed by adding to the system a body force, also singular at contact, e.g., disjoining pressure. As a result, one arrives at a solution and might have an impression that the moving contact-line problem has been resolved "within the classical paradigm" [43]. However, should one add the same body force to the problem without using the 
lubrication approximation, the shear-stress singularity will not be removed. Indeed, the singularity comes from the multivaluedness of the velocity field at the contact line [27], i.e. from the flow kinematics which is a deeper level than dynamics, whilst all what the body force does is changing the pressure distribution. This is sufficient in the lubrication approximation where the issue is with the free-surface shape, i.e. normal-stress boundary condition on the free surface, but this force does nothing to remove the shear-stress singularity in the non-simplified formulation. In other words, the solution obtained in the framework of the lubrication approximation with the no-slip condition and additional body forces is not a uniformly valid approximation of the solution of the original (not simplified) problem, i.e., strictly speaking, it is not a solution.

It should be emphasized that the no-slip condition results in non-integrability of the shear stress only if the contact angle is less than $180^{\circ}$. If it is equal to $180^{\circ}$, and hence the boundary of the flow domain is smooth, albeit with different boundary conditions meeting at the contact line, the solution with no-slip can be found. This has been shown in $[44,45]$, though, sadly, with an error in the sign of the free surface curvature in both papers, as pointed out in [46]. Of course, should the contact angle approach $180^{\circ}$ in a real-life experiment, the viscosity of the displaced fluid (gas) will become increasingly important.

Another point worth a note is that slip reported in molecular dynamics simulations [47-49] should not be confused with slip featuring in the continuum mechanics models. Molecular dynamics simulations refer to the slip occurring on the molecular level without taking the continuum limit, and the often-mentioned 'slip on the scale of several molecules' simply vanishes into zero after the continuum limit is taken. By contrast, slip appearing in the continuum models refers to the velocity on the liquidfacing side of the liquid-solid interface, and the associated length scale is, by definition, macroscopic, not molecular. In continuum mechanics, the 'interface' is a modelling element in its own right, not just a divide between the fluid and the solid. This simple point has been made at length by $\mathrm{H}$. Brenner and Ganesan [50] who appropriately titled their paper 'Molecular wall effects: Are conditions at a boundary "boundary conditions"?'. The answer to this question is 'no, they are not'.

\subsection{Static (equilibrium) contact angle}

The work of Huh and Scriven left without consideration an issue which has later become central to the modelling of dynamic wetting, namely the behaviour of the 'contact angle' formed by the free surface and the solid boundary. If the free-surface shape is not prescribed as in Huh and Scriven's analysis [25], it is subject to the normal-stress boundary condition equating the normal-stress difference across the interface to the capillary pressure, and it needs the value of the contact angle at the contact line confining the free surface as a boundary condition. Unlike the slip condition whose particular form influences the flow only locally, the contact angle has a far-field effect on the free-surface shape and, for small capillary numbers, largely determines it. This aspect becomes particularly important as research in the area moves into micro- and nanofluidics where surface phenomena become dominant.

The static contact angle has been introduced in fluid mechanics by Thomas Young in 1805 [51] as the angle which, in equilibrium, allows the forces acting on the contact line tangentially to the solid surface to be in balance. Mathematically, this balance has the form

$$
\sigma_{1, \mathrm{eq}} \cos \theta_{s}=\sigma_{3, \mathrm{eq}}-\sigma_{2, \mathrm{eq}},
$$

where $\theta_{s}$ is the static (equilibrium) contact angle, $\sigma_{1 \text {,eq }}$ is the equilibrium surface tension of the liquid-fluid interface, $\sigma_{2 \text {, eq }}$ is the equilibrium surface tension of the 

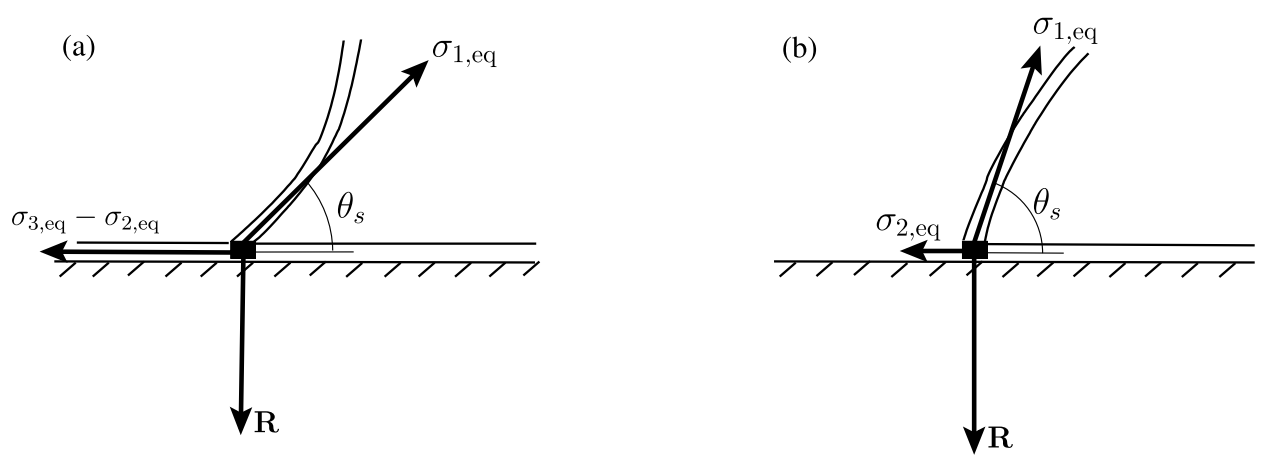

Fig. 1. A sketch illustrating the classical Young equation (1) for a static situation. (a) For a liquid/liquid/solid system, (b) for a liquid/gas/solid system $\left(\sigma_{3, \text { eq }}=0\right)$. The interfaces, which in continuum mechanics are always sharp, are shown here as layers of a finite thickness to emphasize that the surface tensions $\sigma_{2 \text {,eq }}$ and $\sigma_{3 \text {,eq }}$ featuring in (1) come from the thin (molecular scale) layers on the fluid side of the solid-fluid divide whilst the solid is regarded as rigid and serves as a geometric constraint. The contact line is shown as a black box. $R$ is the reaction force acting on the contact line compensating the vertical component of the surface tension acting from the free surface.

interface between the solid and the liquid through which $\theta_{s}$ is measured whilst $\sigma_{3 \text {,eq }}$ is the equilibrium surface tension corresponding to the other fluid-solid interface (Fig. 1a). ${ }^{1}$ Gibbs, who examined Young's argument, explained that $\sigma_{2 \text {,eq }}$ and $\sigma_{3 \text {,eq }}$ are in fact forces per unit length coming from the layers with a thickness on the molecular scale that lie only on the fluid side of the fluid-solid divide, as opposed to the 'full' surface tension coming from such layers adjacent to the fluid-solid divide on both the fluid and the solid side [52] . He even used a special terminology, referring to the 'surface tension' and the 'interfacial tension', to emphasize that, if the solid is regarded as rigid and hence excluded from consideration, it is only the fluid's contribution to the surface tension that counts in the Young equation. Here we will not resurrect this terminological issue and use the term 'surface tension' bearing in mind this distinction.

If one of the fluids is a gas, the corresponding surface tension in (1) is zero (Fig. 1b) as there is no layer made of molecules of the gas on the surface of the solid that would have otherwise produced it.

For the equilibrium state, Young's argument, important as it is, plays only a conceptual role since neither $\sigma_{2 \text {,eq }}, \sigma_{3 \text {,eq }}$ separately, nor even their difference $\sigma_{3 \text {,eq }}-\sigma_{2 \text {,eq }}$ featuring in the Young equation (1) can be measured independently to verify (1). Usually, it is the difference $\sigma_{3 \text {,eq }}-\sigma_{2 \text {,eq }}$ that is inferred from the Young equation itself, with $\theta_{s}$ and $\sigma_{1 \text {,eq }}$ measured independently, but this, obviously, does not validate (1) experimentally. As for its theoretical validation, such works appear from time to time (see, e.g., [53]), but they include more assumptions than Young's equation itself which makes it questionable as to what validates what. In recent years, the Young equation has become the subject of verification via molecular dynamics simulations [54-56], but, again, since the notions of continuum mechanics, including sharp 'interfaces' and the 'contact angles' such interfaces form, are foreign to the molecular dynamics framework and linking them to the simulation data is a nontrivial, not to say tricky, task, the fact that the simulation data can be wrapped up in

\footnotetext{
${ }^{1}$ This force diagram without the reaction force $\mathbf{R}$ and hence with an 'uncompensated' vertical component of the liquid-fluid surface tension reproduced in numerous papers and books makes no sense; it is sufficient to break the system into its elements ('bulk phases', 'interfaces', 'contact line' and consider the equilibrium of each of them [58].
} 
the form of the Young equation (1) should be seen not as a confirmation of the latter but as a partial validation of the molecular simulation technique. The bottom line here is that, although the Young equation (1) occasionally causes misunderstanding and an exchange of opposite views [57,58], as rightly pointed out in [6], it does not need justification nor validation as it is ultimately just a balance of forces, Newton's second law applied to the contact line as an element in the continuum mechanics modelling of a system with interfaces.

Thus, in statics, one can either specify $\sigma_{3, \text { eq }}, \sigma_{2 \text {,eq }}$ (or just their difference) and calculate $\theta_{s}$ from (1) or, equivalently and more conveniently from a practical viewpoint, simply prescribe $\theta_{s}$ as an independently measurable material constant specific to the three contacting media. It is implied, of course, that the solid substrate is 'ideal', i.e., smooth chemically homogeneous rigid and inert, so that the static contact angle is specified uniquely. Non-ideal substrates, where one has to deal with the contact-angle hysteresis, form a special chapter in the studies of wetting [59-61], and we will leave this issue aside for the time being and return to it in Section 5.

\subsection{Dynamic contact angle}

Experiments have shown long ago $[62,63]$ that the optically observed contact angle in a situation where the contact line moves across a solid surface is not the same as in equilibrium. Improvements in the spatial resolution of the measurements only confirmed this fact so that it had to be accounted for in theoretical models.

Regarding the contact angle, the logic of all mathematical models (with a single exception to be discussed later) has been as follows. Since the dynamic contact angle deviates from the static one and varies with the contact-line speed, one can specify it as a function of the contact-line speed and, of course, material constants characterizing the contacting media. This function then becomes part of the mathematical model, an input into it, and, for a given liquid/fluid/solid system, it is the same for all types of flow involving moving contact lines.

The problem is then to find this function, i.e., if $\theta_{d}$ is the dynamic contact angle featuring in the continuum mechanics description and $U$ is the contact-line speed, then one has to find

$$
\theta_{d}=f\left(U, \theta_{s}, k_{1}, k_{2}, \ldots\right),
$$

where $k_{1}, k_{2}$, etc are material constants characterizing the contacting media. For the equilibrium state, $(2)$ should recover the static contact angle, i.e., $f\left(0, \theta_{s}, k_{1}, k_{2}, \ldots\right)=$ $\theta_{s}$.

Prompted by the set of standard experiments (see, e.g., Ch. 3 of [10] for a review), where an increase of the measured contact angle is associated with an increasing contact-line speed, the function in (2) is suggested to be invertible, so that the velocity-dependence of the dynamic contact angle is often sought in an equivalent form

$$
U=F\left(\theta_{d}, \theta_{s}, k_{1}, k_{2}, \ldots\right)
$$

The first functions put into full-scale continuum models had been chosen primarily for their simplicity and mathematical convenience rather than on the basis of some physical justification. However, they broadly reflected the expected tendency of the contact angle to increase with the contact-line speed and were taken in the form of a power law

$$
U=k_{1}\left(\theta_{d}-\theta_{s}\right)^{k_{2}}
$$


with $k_{2}=1$ [31], $k_{2}=2$ [64] and $k_{2}=3[64,65]$. Here we mention only representative papers that used (4); more references can be found in Chap. 3 of [10]. Experimentalists, who consider (4) with $k_{2}=1$, refer to $k_{1}$ as the 'moving contact-line mobility' [66].

Blake and Haynes [67] proposed a more sophisticated expression for the dynamic contact angle as a function of the contact-line speed which came to be known as the molecular-kinetic theory (MKT). The corresponding formula is given by

$$
U=2 K \lambda \sinh \left(\frac{\lambda^{2} \sigma_{1, \mathrm{eq}}\left(\cos \theta_{s}-\cos \theta_{d}\right)}{2 k T}\right),
$$

where constants $\lambda$ and $K$ characterize molecular motion on the substrate (the length between adsorption sites and the molecular jump frequency, respectively), $k$ is the Boltzmann constant and $T$ is the absolute temperature. A strong point of (5) is that, unlike power-law dependencies (4), it is physically motivated and has been extensively used in molecular dynamics simulations [68,69], providing the language for interpreting the simulation data. At the same time, from the perspective of the continuum mechanics modelling, the MKT is conceptually no different from (4) as it also proposes a velocity-dependence of the dynamic contact angle in the form of (3) and this dependence is to be used as an input in an embracing continuum model. Additionally, such a model will require a slip boundary condition to remove non-integrability of the shear stress at the contact line as described above.

A somewhat unexpected development of the MKT emerged with the notion of the 'moving contact-line friction' [35,70-72]. Its idea is that, if the contact line moves, in the classical Young equation (1) there must appear a 'friction force' $F_{\mathrm{fr}}(U)$ :

$$
\sigma_{1, \text { eq }} \cos \theta_{d}=\sigma_{3, \text { eq }}-\sigma_{2, \text { eq }}-F_{\text {fr }}(U)
$$

If, in the simplest case, the moving contact-line friction is assumed to be proportional to the contact-line speed, $F_{\text {fr }}(U)=\mu_{c l} U$, then, after using this expression in (6) and subtracting (6) from (1), one arrives at

$$
\cos \theta_{s}-\cos \theta_{d}=\frac{\mu_{c l}}{\sigma_{1, \mathrm{eq}}} U .
$$

This formula, which is at the core of the models in [35,70-72], results from a fundamental misconception. It presumes that the contact-line motion is caused by the so-called 'unbalanced Young stress' $\sigma_{1 \text {,eq }}\left(\cos \theta_{s}-\cos \theta_{d}\right)$ whilst the solid resists this motion via friction. This widely held view is best illustrated by a quote from [73]:

"When a liquid droplet contacts a solid substrate, the unbalanced capillary force, $\sigma_{1, \text { eq }}\left(\cos \theta_{s}-\cos \theta_{d}\right)$, drives the liquid to spread on the substrate until it reaches equilibrium".

In other words, the deviation of $\theta_{d}$ from $\theta_{s}$ is regarded as the cause of motion whilst in Young's equation, which is nothing but Newton's second law applied to the contact line, it is the forces (i.e. surface tensions) that dictate what the contact angle must be for these forces acting on the contact line with negligible mass to be in balance. Then, should Young's (and Newton's) logic be followed, one would have to conclude that in (6) it is the friction force that drives the motion. Despite this obvious mistake, the idea of the 'contact line friction' attracted some following, with an attempt to justify it using molecular dynamics simulations [74], its derivation as a sharp-interface limit of the phase-field model [75] and a considerable effort invested in its numerical implementation [76,77]. What is important for us here is that, ultimately, equation (7), erroneous as it is, is nothing but another formula of the form (3). 


\section{4 'Apparent' contact angle}

Early works on moving contact lines [29,78-80] assumed that

$$
\theta_{d} \equiv \theta_{s}
$$

On the one hand, this bold assumption removes the need for inventing a nontrivial function featuring in (2) and, relying on the classic Young equation (1) and nearly two centuries of its appreciation, looks very plausible. On the other, however, it poses an obvious problem of how to reconcile it with the undeniable fact that the dynamic contact angle observed in experiments is nowhere near the static (equilibrium) value. This dilemma has led to a peculiar twist in the development of the dynamic wetting theory which we will outline.

As all measurements, measurements of the contact angle, usually optical, have limited accuracy. Their accuracy depends on many factors $[5,81]$ including, in particular, (i) how the free surface is visualized, (ii) spatial resolution of the optical device, (iii) the fitting algorithm used to create, locally or globally, a sharp interface from the free-surface visualization so that one could determine the angle this interface forms with the substrate, and (iv) vibrations in the system [81] blurring the picture. As a result, the measured value $\theta_{\exp }$ has certain margins of error which accumulate the combined effect of all these factors. The angle $\theta_{d}$ featuring in the models is expected to describe the angle $\theta_{\exp }$ observed in experiments or, equivalently, the measured angle $\theta_{\exp }$ is meant to verify experimentally that $\theta_{d}$ provides an accurate description of what is observed in reality, thus validating the theory. If the theory is correct, then the better the accuracy of the experiments the closer $\theta_{\exp }$ is to $\theta_{d}$. In short, experiment is meant to measure $\theta_{d}$, and if the discrepancy between $\theta_{d}$ and $\theta_{\exp }$ is beyond the margins of error, then it is the theory responsible for $\theta_{d}$ that has to be revised. This is how the basic methodology of physical experimentation and its interaction with theory work and help to improve the theory, and one would have expected it to be followed.

However, the assumption (8) put into continuum models back in the 1970s despite its obvious conflict with all known experiments prompted a fundamental departure from how theory and experiment normally interact. In order to reconcile (8) with experiments, theoreticians of the 1970s proclaimed that what experimentalists measure, accurately or not so accurately, is not $\theta_{d}$ but some 'apparent' contact angle $\theta_{\text {app }}$ whilst $\theta_{d} \equiv \theta_{s}$ as (8) demands.

An obvious advantage of this line of reasoning is that the discrepancy between theory responsible for $\theta_{d}$ and experiment no longer matters and requires no revision of the theory. All what one needs to do now is to invent a suitable artificial construct - the apparent contact angle - to interpret what experiments observe. Thus, research into the behaviour of $\theta_{d}$ has been tacitly replaced with research into what $\theta_{\text {app }}$ might be.

In experiments, the accuracy of measuring $\theta_{\exp }$ depends on a multitude of factors entangled in a complex way $[5,81]$ whilst the mathematical description of the corresponding flow is precise and contains no other angles apart from $\theta_{d}$. Attributing the deviation of $\theta_{\exp }=\theta_{\text {app }}$ from $\theta_{d}$ to bending of the free surface by viscous stresses near the contact line $[78,80], \theta_{\text {app }}$ is defined as simply an angle in the mathematical description of the free surface between the tangent to it at some distance either from the substrate or from the contact line and the solid boundary (Fig. 2). This approach unavoidably introduces an adjustable parameter, namely the ratio of this distance to some lengthscale featuring in the model and characterizing either where the free surface is supposed to be unaffected by the motion [78] or the slip length [80]. Interestingly, although the spatial resolution of experimental measurements is gradually 

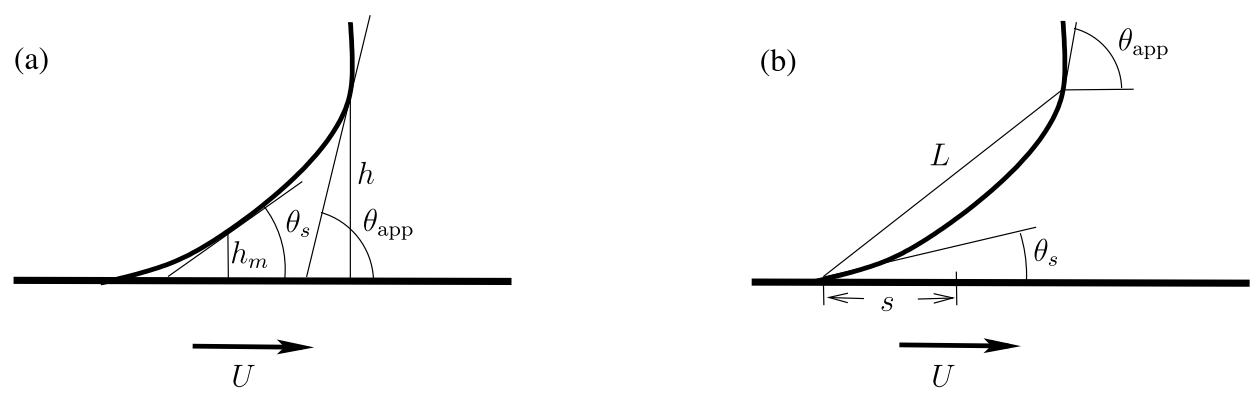

Fig. 2. A definition sketch for the 'apparent' contact angle $\theta_{\text {app }}$ introduced (a) by Voinov [78] and (b) by Cox [80]. The static (equilibrium) contact angle $\theta_{s}$ in [78] is measured at some distance $h_{m}$ from the substrate/contact line with the region between this cross-section and the contact line excluded from consideration. In [80], $\theta_{s}$ is the actual contact angle featuring in the continuum mechanics formulation, $s$ is the slip length. The 'apparent' contact angle is assumed to be measured either at height $h$ from the substrate [78] or at distance $L$ from the contact line [80].

improving, with the latest results claiming it to be in the nanoscale range $[82,83]$, the bending responsible for deviation of the apparent contact angle from $\theta_{d}$ is somehow always below it.

The first expression relating the apparent contact angle with the contact-line speed was proposed by Voinov [78], who derived, in a mathematically rather crude way, the equation

$$
\theta_{\mathrm{app}}=\left[\theta_{s}^{3}+\frac{9 \mu U}{\sigma_{1, \mathrm{eq}}} \ln \left(\frac{h}{h_{m}}\right)\right]^{1 / 3}
$$

where $\mu$ is the fluid's viscosity, $h$ is the distance from the substrate to the point on the free surface where the apparent angle $\theta_{\text {app }}$ is measured (Fig. 2a) and $h_{m}$ is some microscopic distance, estimated by Voinov to be in the range $\sim 10^{-6}-10^{-7} \mathrm{~cm}$, where the contact angle, although defined in the same manner as the apparent one, for some reason has its equilibrium value $\theta_{s}$ (Fig. 2a). The flow in the region between the contact line and the cross-section where $h_{m}$ is located is not considered, so that in essence $h_{m}$ is a 'cut-off' length. The notion of a 'cut-off' is rather popular (see, e.g., [84]) as it leaves the difficulties out of consideration but, of course, it has no place in mathematics where the operation of cutting off regions and/or modelling difficulties is not defined.

As one can see, equation (9) has the same functional form as (2) with $\theta_{d}$ replaced by $\theta_{\text {app }}$ and an additional adjustable parameter $h / h_{m}$, i.e.,

$$
\theta_{\text {app }}=\phi\left(U, \theta_{s}, \frac{h}{h_{m}}, k_{1}, k_{2}, \ldots\right) .
$$

Eggers and Stone [85] suggested a modification of Voinov's formula (9) in the case of perfect wetting $\left(\theta_{s}=0\right)$ by making $h_{m}$ velocity dependent. The end result again takes the form of $(10)$.

Cox [80] developed the approach of Voinov and, defining the apparent contact angle as measured at distance $L$ from the contact line (Fig. 2b), derived a relationship between $\theta_{\text {app }}$ and $U$, which also accounted for the viscosity of the displaced gas, in 
the form

$$
g\left(\theta_{\mathrm{app}}, k_{\mu}\right)=g\left(\theta_{s}, k_{\mu}\right)+\frac{\mu U}{\sigma_{1, \mathrm{eq}}} \ln \left(\frac{L}{s}\right)
$$

where

$$
\begin{gathered}
g\left(\theta, k_{\mu}\right)=\int_{0}^{\theta} \frac{d \theta}{f\left(\theta, k_{\mu}\right)}, \\
f\left(\theta, k_{\mu}\right) \equiv \frac{2 \sin \theta\left\{k_{\mu}^{2}\left(\theta^{2}-\sin ^{2} \theta\right)+2 k_{\mu}\left[\theta(\pi-\theta)+\sin ^{2} \theta\right]+\left[(\pi-\theta)^{2}-\sin ^{2} \theta\right]\right\}}{k_{\mu}\left(k_{\mu}^{2}-\sin ^{2} \theta\right)[(\pi-\theta)+\sin \theta \cos \theta]+\left[(\pi-\theta)^{2}-\sin ^{2} \theta\right](\theta-\sin \theta \cos \theta)},
\end{gathered}
$$

$s$ is the length of the slip region and $k_{\mu}$ is the gas-to-liquid viscosity ratio. Mathematically, this relationship is of the form

$$
\Phi\left(U, \theta_{\text {app }}, \theta_{s}, \frac{L}{s}, k_{1}, k_{2}, \ldots\right)=0
$$

which can be resolved into the explicit form of (10) though the function $\phi$ will then have to be found numerically.

The assumption that $\theta_{d} \equiv \theta_{s}$ used by both Voinov's and Cox's formulae can, of course, be replaced by any velocity-dependence of $\theta_{d}$ with the end result still taking the form of (10). An example of this synthetic modelling can be found in the work of Petrov and Petrov [86], who combined Voinov's formula for the apparent angle (9) with the molecular-kinetic theory of Blake and Haynes (5) ending up with an expression

$\theta_{\text {app }}=\left\{\arccos ^{3}\left[\cos \theta_{s}-\frac{2 k T}{\lambda^{2} \sigma_{1, \mathrm{eq}}} \ln \left(\frac{U}{2 K \lambda}+\sqrt{\left(\frac{U}{2 K \lambda}\right)^{2}+1}\right)\right]+\frac{9 \mu U}{\sigma_{1, \mathrm{eq}}} \ln \left(\frac{h}{h_{m}}\right)\right\}^{1 / 3}$,

which is again in the form of (10).

The last formula for the apparent contact angle to be mentioned here simply for completeness is limited to thin films and came to be known as "Tanner's law" [87]. Tanner considered a particular quasi-steady flow where a thin drop spreads over a solid surface already covered by an even thinner 'precursor' film. Then, as one can easily imagine, the drop will have a bell-shaped profile with an inflection point, and Tanner found that the angle $\theta_{\text {app }}$ between the tangent to the free surface at this point and the solid boundary is related to the speed $U$ with which this inflection point moves by

$$
U=k_{1} \theta_{\text {app }}^{3}
$$

and calculated the coefficient $k_{1}$ in terms of the parameters of the problem. Unlike Voinov's and Cox's formulae, (14) does not involve any adjustable parameters; the point where the apparent contact angle $\theta_{\text {app }}$ is supposed to be measured is specified exactly and the value of $k_{1}$ is also given. Perhaps, it is these attractive features together with the simplicity of equation (14) that elevated it to the status of a 'law' and inspired considerable following (e.g. [88-90]). However, in reality "Tanner's law" 
is just another formula relating $\theta_{\text {app }}$ and $U$ for a particular problem, a particular case of Voinov's formula (9) with $\theta_{s}=0$ or a particular case of the power law (4) with $\theta_{d}$ replaced by $\theta_{\text {app }}$ and $\theta_{s}=0, k_{2}=3$.

\subsection{Comparison with experiment: agreement and question marks}

The multitude of functions proposed to describe the velocity-dependence of the contact angle attracted considerable experimental effort aimed at singling out the 'right' one. The conventional approach to testing different formulae describing the dynamic contact angle as a function of the contact-line speed is to use one of the standard experimental configurations [1] and, treating the parameters in the formulae as adjustable, fit the curves these formulae produce to the data, often employing statistical analysis to improve the fitting. This approach in its quintessential form has been put in practice by Seveno et al. [91] who examined the molecular-kinetic theory of Blake and Haynes (5), Voinov's 'hydrodynamic model' (9), Petrov and Petrov's combined formula (13) as well as a more recent formula of de Ruijter et al. [92], which was derived specifically for the late stage of the drop spreading, features a mathematically unjustifiable 'cut-off' length and also has the familiar form of (10). Seveno et al. concluded that

"despite the range of the data and the sophisticated and statistically robust method offered by G-Dyna [the software used to process the data], the results illustrate the difficulty in defining which model is most appropriate by curve fitting".

In other words, for the rather limited range of the contact angles tested and within the accuracy of the experiments, all of the formulae agree with the data reported in [91] satisfactorily so that one cannot decide conclusively in favour of one of them nor rule out any of them. Other experiments performed to test the models in the conventional way broadly support this conclusion [5]: with the appropriate choice of constants, standard experiments can be satisfactorily described, almost invariably in terms of an apparent contact angle or with the use of an ad hoc formula. Thus, standard experiments do not allow one to discriminate between models nor can one use the contact angle data to find more about the physics of dynamic wetting and further develop its mathematical theory.

However, the formulae listed above, albeit broadly describing the same trend an increase of the contact angle as the contact-line speed increases - produce slightly different curves in the contact angle-versus-speed plane so that, with improving accuracy of experimentation, one might be able to discriminate between them. This hope motivated further research.

Following a hypothesis discussed in [93] that different formulae might correspond to different regimes of dynamic wetting, Karim et al. [94] compared experimentally 'spontaneous' and 'forced' spreading of the same liquid on the same solid and reported that the resulting curves in the contact angle-versus-speed plane were distinctly different. However, instead of questioning the very approach of describing the dynamic contact angle as a preset function of the contact-line speed which purports to cover all regimes, the authors attempted to reconcile this approach with what they observed. After trying to fit the molecular-kinetic theory of Blake and Haynes (5) and the purely hydrodynamic formula of Voinov (9) to their data, they concluded that the MKT is better at describing the data for forced spreading whilst Voinov's hydrodynamic formula fits better the data for spontaneous spreading. Karim et al. even discussed 'rules of thumb' for an experimentalist to follow which "involve a priori knowledge of the expected behaviour". 
The results of Karim et al. [94] cast serious doubt on the validity of the very approach where the dynamic contact angle is described as a function of the contactline speed since the terms 'spontaneous' and 'forced' spreading are nothing but descriptive labels of the extreme situations whilst one can have numerous flows between these two extremes with the elements of 'spontaneous' and 'forced' behaviour mixed in different proportions. Various regimes of sloshing are but one example. As for the rules of thumb regarding application of different formulae based on a priori knowledge of what to expect, these rules essentially give up on any predictive power of the models in question and look simply like an attempt to save the approach which is falling apart in the light of experiments with improved accuracy.

An even more serious doubt on the approach that led to all the formulae mentioned above has been cast by the experimentally discovered effect of 'hydrodynamic assist of dynamic wetting'. The essence of this effect is that the measured dynamic contact angle for a fixed contact-line speed is not fixed; it can be varied by varying the flow field/geometry near the moving contact line. This effect, first observed for air entrainment in curtain coating [95] (hence its technologically sounding name), was later reported, also in the context of curtain coating, for the contact angle itself $[96,97]$.

The origin of hydrodynamic assist in curtain coating regarding the postponement of air entrainment remains a matter of ongoing research focussing on the role of the displaced air [98]. As for the contact angle behaviour, it has been shown conclusively by Wilson et al. [99] that, no matter how the apparent contact angle is introduced, the effect cannot be described by slip models even if both the slip length and the actual contact angle are used as adjustable parameters, i.e., for all possible functions that would describe the velocity dependence of the contact angle. Admittedly, Wilson et al. did not consider the role of the displaced air, so this direction of research awaits completion, though one would note that the role of air diminishes for smaller contact angles, i.e., where the 'assist' as such is observed.

An effect that qualitatively also belongs to 'hydrodynamic assist' was reported by Bayer and Megaridis [100] who experimentally investigated the impact of drops on a solid substrate, applied Blake and Haynes (5) and Cox (11) formulae and concluded that "even for a single liquid there is no universal expression to related contact angle with contact line speed".

Thus, improvements in the accuracy of experiments and the focus on different flows as well as on the role played by hydrodynamic factors other than the contactline speed cast doubt on the adequacy of the very approach that produced the formulae mentioned above. At the same time, one can always argue that these quantitative experiments are suggestive rather than conclusive as the physical factors not accounted for in the models might be at play and the accuracy of the measurements can always be questioned. What is needed at this point is a decisive argument that would by-pass these lingering doubts and become universally understood and accepted, similar to the qualitative argument that drew a line under Galileo's experimentation with the balls of different mass he was pensively dropping from the Tower of Pisa.

\subsection{Different functions, the same approach}

From the viewpoint of the mathematical structure, the models/theories reviewed above, despite their apparent diversity, represent the same modelling approach. In this approach, the velocity-dependence of the dynamic contact angle, actual or apparent, is an input in the model, the same for all flows involving moving contact lines. The natural questions that arise at this point are as follows: 
- Should the velocity dependence of the dynamic contact angle be an input in a model of dynamic wetting in principle?

- Can we suggest a class of flows which would settle the above question regardless of such issues as the accuracy of measurements and/or physical factors additional to dynamic wetting?

Below, we answer these questions and examine the validity of the very approach where the contact angle is an input into the model prescribed as (2), or (3), or (10), or even (12).

\section{A qualitative 'litmus test': can we discard a model at a glance?}

The view that the dynamic contact angle is a function of the contact-line speed is shared by the vast majority in the dynamic wetting community and beyond. In order to test it, we need an experiment which could be understood on a qualitative level irrespectively of such issues as the accuracy of measurement or whether the angle in question is actual or apparent.

Consider a drop gently placed onto a smooth inert chemically homogeneous solid substrate (Fig. 3). Initially, the contact angle is $180^{\circ}$, which is simply a mathematical fact as there are two smooth surfaces touching. Once pressed against the substrate, e.g. by gravity, the drop will form an infinitesimally small area of contact with the solid and this will trigger its spreading over the substrate. Thus, the motion begins from rest $(U=0)$ and from the contact angle of $180^{\circ}$ (or infinitesimally close to it). This starting point is shown as (a) in the contact angle-versus-speed plane of Figure 3.

Eventually, the drop's spreading will come to an end, and we again have zero contact-line speed whilst the contact angle now has its static (equilibrium) value, see Figure 3c.

In the process of spreading, both the contact angle and the contact-line speed vary, and in the angle-versus-speed plane this corresponds to some continuous curve connecting the starting and the end points, (a) and (c) in Figure 3. This curve cannot go along the vertical axis as otherwise there would be no spreading. Qualitatively, a curve connecting these points is shown in Figure 3, where (b) refers to the whole curve except the start and the end points. The reader is invited to think of and draw a curve of his/her own, but, no matter what curve one chooses to draw, even the one corresponding to the inertial regime where the contact line overshoots its equilibrium position and has to come back, it is already clear that the dynamic contact angle is not a function of the contact-line speed. Indeed, as one can see, for every value of the contact-line speed under the curve in Figure 3 there are two values of the contact angle. This completely invalidates the very approach of having the velocitydependence of the dynamic contact angle, actual or apparent, as an input in any realistic mathematical model of dynamic wetting, i.e., undermines (2) and (10) in principle.

It is important to emphasize also that the upper branch of the curve shown in Figure 3, from the starting point (a) to the turning point, shows that the contact angle decreases as the contact-line speed increases. This is contrary to what all formulae proposed for the contact angle, actual or apparent, predict. Experiments dealing with the late stages of the drop spreading, e.g. [91,94], missed this behaviour.

At this point, a reader without a background in mathematical modelling but keen on defending the approach outlined in Section 2 is bound to argue that, as a drop touches a substrate at what in macroscopic terms is a point, there is not yet any contact line as such and hence one should not attribute any speed to it nor extract a contact-angle value from the initial configuration. Although for those 


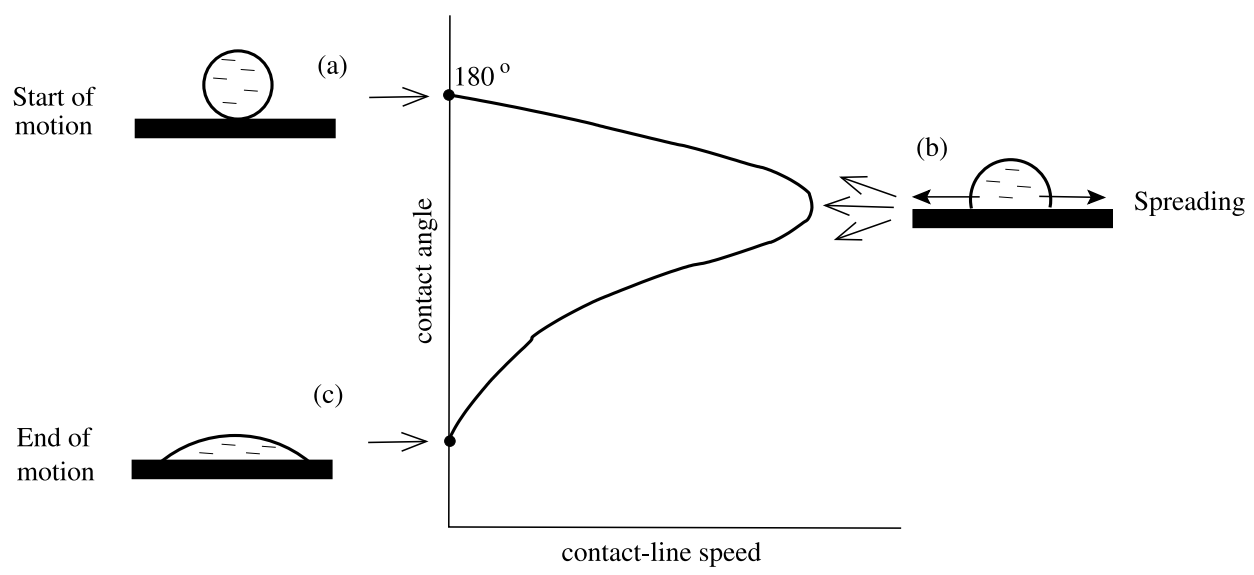

Fig. 3. A qualitative diagram in the contact angle-versus-speed plane for a drop spreading from rest. The start of motion corresponds to the drop touching the solid (a) whilst the final state is the drop in equilibrium (c). The process of spreading (b) corresponds to a continuous curve connecting (a) and (c): the contact line accelerates and then decelerates whist the contact angle is going down. Then, for the same values of the contact-line speed under the curve one will have two different values of the contact angle. Hence the contact angle is not a function of the contact-line speed. This completely undermines the very approach of using the dynamic contact angle as a function of the contact-line speed as an input in mathematical models of dynamic wetting.

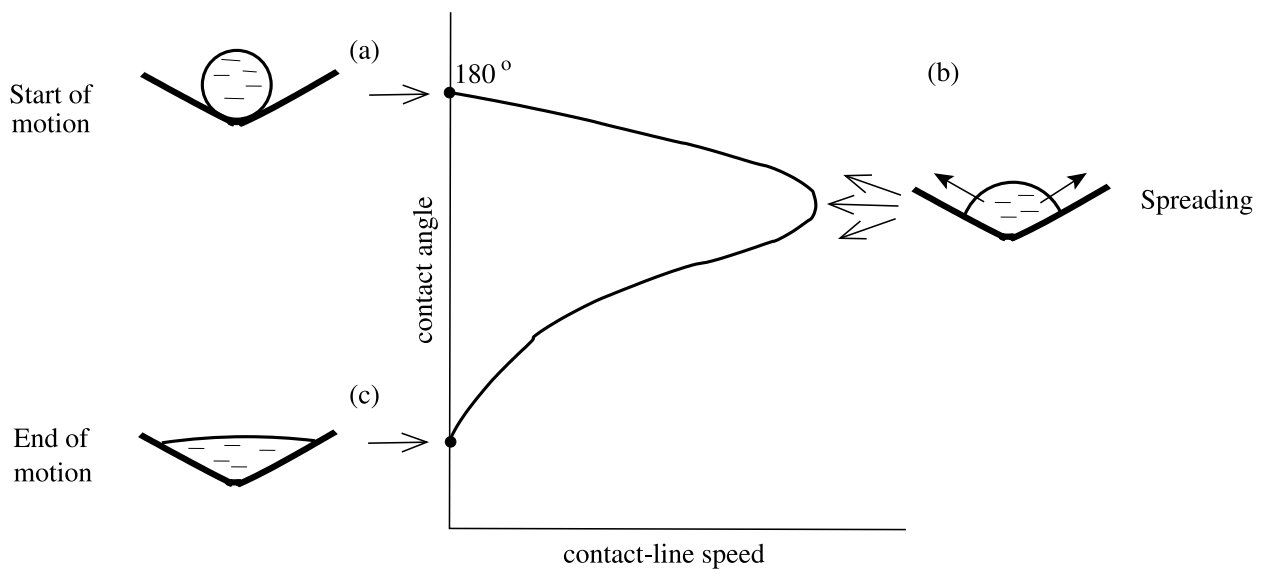

Fig. 4. A variation on the situation sketched in Figure 3. Now the drop has a finite contact area and a finite-length contact line from the start. Then, one does not need a continuity argument, as in the case of Figure 3, to ascribe the values to the contact-line speed and the contact angle at the onset of motion. The initial contact-line speed is zero and the contact angle, with the correspondingly profiled substrate, is $180^{\circ}$. The rest of the argument is the same as for Figure 3.

familiar with mathematical modelling this kind of objection is removed by a simple continuity argument, the readers who are far from mathematics are best addressed by referring them to Figure 4 sketching a drop deposited onto and spreading over a profiled substrate. In this setting, the base of the drop is finite and a finite-length contact line is present from the start. The rest of the argument is the same as for the flow shown in Figure 3. 


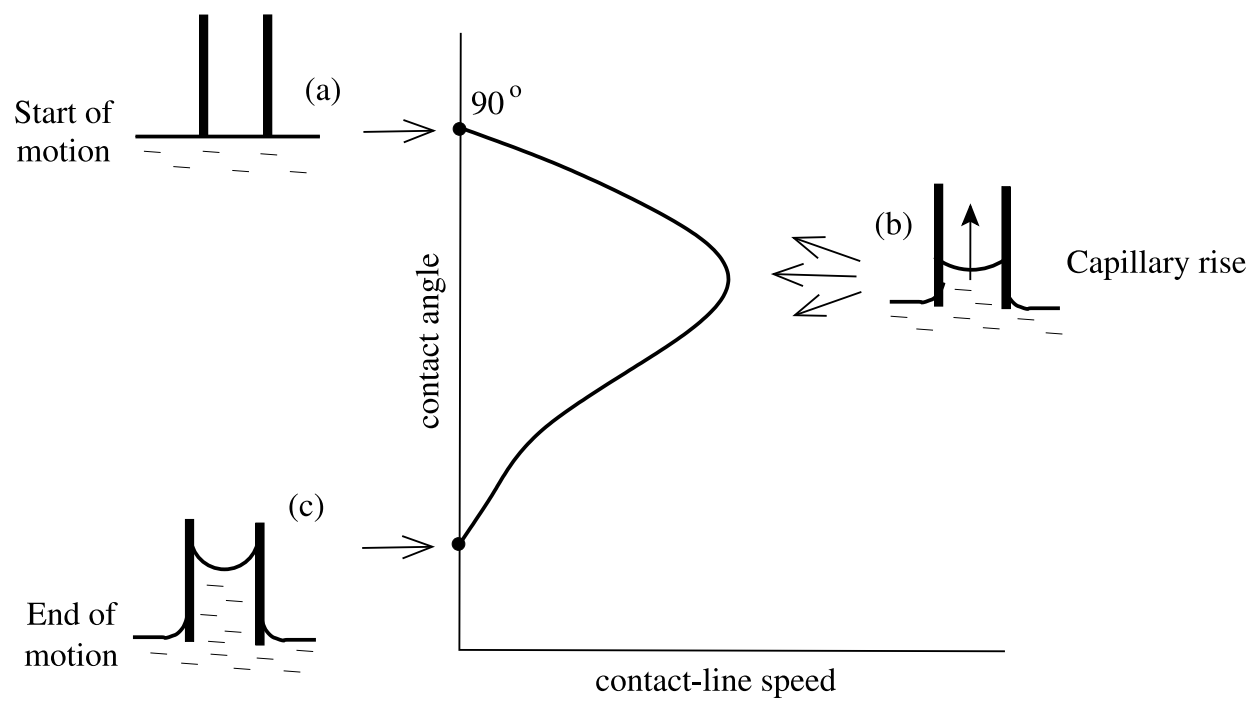

Fig. 5. A sketch illustrating the contact angle-versus-speed diagram for the capillary rise. Qualitatively, it is similar to that of the spreading drop Figure 3. The motion starting from rest (a) ends with the meniscus reaching equilibrium (c). The start of motion and the end of motion correspond, by definition, to zero contact-line speed. Then, in the process of capillary rise the contact-line speed will, first, increase and then go down whilst the contact angle will be monotonically decreasing. We have again that the contact angle is not a function of the contact-line speed and there is no reason to expect that any part of the angle-versus-speed diagram for the capillary rise will coincide with that for the spreading drop, though they may intersect or be close as the contact angle approaches its static value (the same for the same liquid/fluid/solid system).

The contact angle behaviour similar to that shown schematically in Figures 3 and 4 follows also from another flow configuration, namely the capillary rise from rest, see Figure 5. In this case, we have a pool of liquid with the free surface touched from above by a capillary tube (Fig. 5a). Then, the meniscus will appear and start rising into the capillary. The motion again starts from rest $(U=0)$ and the initial contact angle is $90^{\circ}$ whilst when the meniscus reaches its equilibrium position, where the capillary driving force is balanced by gravity, the flow will come to a halt $(U=0)$ and the contact angle reach its static (equilibrium) value $\theta_{s}<90^{\circ}$ (Fig. 5c). Then, as before we have a downward curve (b) connecting (a) and (c), and, as in the case of the spreading drop, this curve cannot go along the vertical axis as otherwise there would have been no capillary rise at all. Thus, we again have an accelerating contact line with the decreasing dynamic contact angle at the start of the process and the decelerating contact-line together with the decreasing contact angle towards the end of it.

The two curves sketched in Figures 3 and 5 start from different points, so that obviously they do not coincide. This completely rules out the functional form (3) as an alternative to (2) and a potential universal input into any theory of dynamic wetting.

A flow configuration which could be convenient in physical and especially molecular-dynamics experimentation for a systematic study of spontaneous spreading is shown in Figure 6 . If the free surface of a liquid is touched by a wedge-shaped solid or, for axial symmetry, by a cone, the initial state is far from equilibrium so that the contact line will start moving from rest (Fig. 6a), accelerating until its speed 


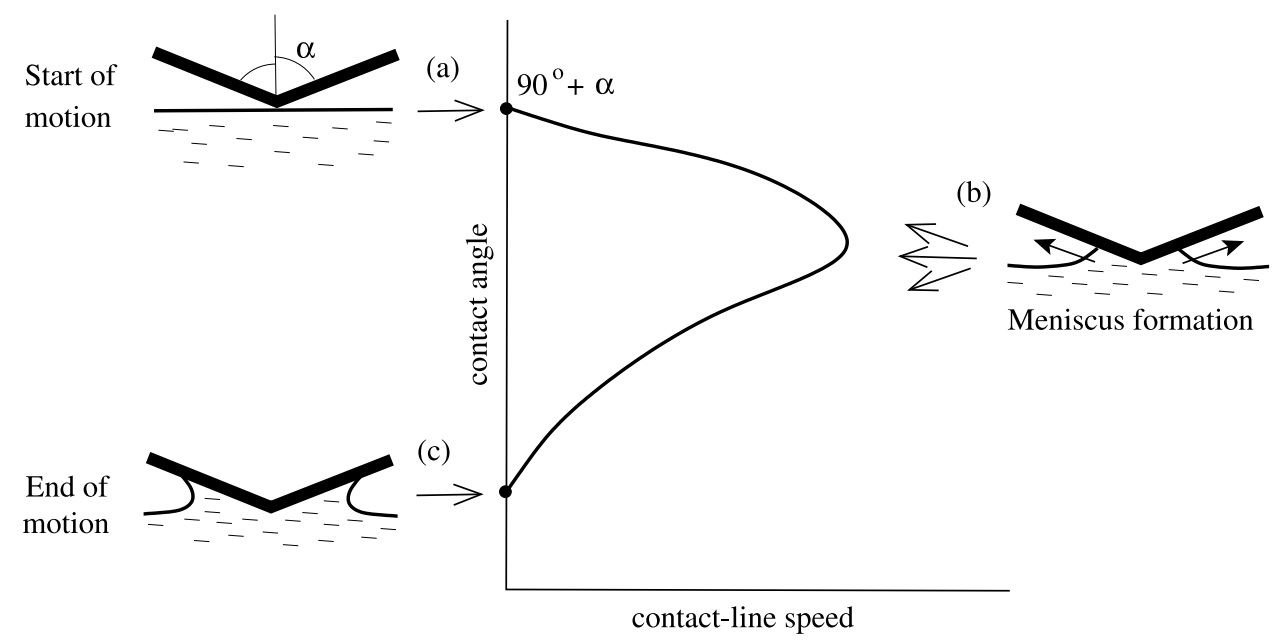

Fig. 6. A sketch showing the contact angle-versus-speed diagram for a meniscus forming as a pool of liquid is touched by a wedge-shaped or conical solid. The flow again starts from an out-of-equilibrium initial position and the angle-versus-speed diagram has qualitatively the same shape as in Figures 3 and 5. This configuration has an advantage for physical and molecular dynamics experimentation as the opening angle of the wedge/cone makes it possible to obtain a one-parametric family of angle-versus-speed curves which could be used for comparison with the results produced by mathematical models.

reaches a certain maximum value (Fig. 6b) and then it will decelerate as the system approaches its final equilibrium state where the meniscus is at rest and the contact angle is the static one (Fig. 6c). In this flow geometry, the opening of the wedge/cone is a free parameter which can be varied from one experiment to the next, allowing one to generate a one-parametric family of curves in the angle-vs-speed plane starting from different points on the vertical axis. An immediate consequence of this is that even the general form of the velocity-dependence of the apparent contact angle (12), which could, potentially, include the case of several contact angles for the same contact-line speed, and the same form for the actual contact angle, are ruled out as a universal input into a theory in principle.

Now, we can see the general picture. For any spontaneous spreading of a liquid over a solid surface, or indeed a flow with, so to speak, an element of spontaneity, i.e., any flow apart from the forced spreading at a fixed contact-line speed, the contact angle varies in a way specific to the flow configuration. The corresponding path in the contact angle-versus-speed plane can be regarded as a 'signature' of the particular spreading process. Even the four flow configurations considered here allow us to assert that there is no universal relationship between the contact angle, actual or apparent, and the contact-line speed even in the generic form (12), not to mention explicit relationships (2), (3) or (10). Thus, the entire direction of research aimed at finding such a relationship is a dead end. As one can see, all models outlined in the previous section belong to this dead-end direction.

We can now formulate a 'litmus test' allowing one to assess mathematical models at a glance.

'Litmus test': if a mathematical model of dynamic wetting has the velocity-dependence of the dynamic contact angle, actual or apparent, as an input, thus presuming the same curve in the angle-versus-speed plane irrespective of the flow, then this model will fail to describe, even 
on a qualitative level, a multitude of capillary flows and, as a contribution to theoretical fluid mechanics, does not deserve further attention.

It is instructive to have a look back at the overview of models given in Section 2 to see where the logic of their inception and development went wrong. The statement that 'since the dynamic contact angle deviates from the static one and varies as the contact-line speed varies, then it must be a function of the contact-line speed' gives the gist of the argument behind all these models, and it is at this point that the development is sent down the wrong path. Indeed, the fact that the dynamic contact angle and the contact-line speed both vary does not mean that one is a function of the other. They can be both varied by an underlying physical process and varied in different ways depending on a particular flow configuration which brings in this physical process.

Perhaps, a contributing factor to this initial confusion was that the original moving contact-line problem had been introduced by Huh and Scriven [25] using a simplified problem formulation where it appeared as 'the problem of non-integrable shear-stress singularity'. If looked at through the prism of a general formulation, the problem appears as the problem of non-existence of a solution in the framework of the conventional model [101]. The former formulation calls for a recipe to make shear stress integrable and, once this is fixed, for a recipe to make the contact angle vary whilst the latter indicates that some essential physics is missing and, once incorporated into a model, it should sort out all its aspects.

We should note here that the models failing our 'litmus test' do not contribute to science, i.e., to theoretical fluid mechanics, but in particular engineering applications, where the role of the flow's 'spontaneity' is small, some of these models can still be useful as a simple way to capture the main effect of the moving meniscus on the overall flow.

\section{Moving contact lines on an ideal substrate: resolution of the problem}

As discussed in [7], there are three holistic frameworks one can use to describe a fluid flow in general and the dynamic wetting process in particular, namely (a) molecular dynamics simulations, (b) statistical physics, e.g. in the form of the lattice Boltzmann modelling, and (c) continuum mechanics. Each of these frameworks has its own toolkit of basic elements into which the model must be cast. The original 'moving contactline problem' and the 'dynamic contact angle problem' that emerged from it both belong to the continuum mechanics framework as only there one has 'contact lines' and 'contact angles' as the lines and angles formed at the intersection of sharp 'interfaces' confining 'bulk phases' where partial differential equations describing moving continua operate. Other frameworks need special constructs to relate their findings to these continuum concepts which are foreign to them.

\subsection{Requirements for the mathematical model of dynamic wetting}

The required mathematical model of dynamic wetting formulated in the framework of continuum mechanics should have the following properties:

(i) 'Litmus test'. The model should pass our 'litmus test', i.e., have the dynamic contact angle $\theta_{d}$ not as an input but as an outcome, as part of the solution, and for different flows it should produce different paths in the contact angle-versusspeed plane, as suggested by the spontaneous spreading flows considered in the 
previous section. In particular, it should be able to describe the contact angle decreasing as the contact line accelerates.

(ii) Qualitative features. The model should describe other known qualitative features of the dynamic wetting flow, such as the 'rolling' kinematics and the effect of 'hydrodynamic assist of dynamic wetting'.

(iii) Quantitative comparison with experiments. The model should be able to describe quantitatively with acceptable accuracy the velocity-dependence of the dynamic contact angle measured in standard experiments. This requirement should be taken with caution and applied thoughtfully, given that it is very difficult to ensure that the substrate is 'ideal' or, if there is a discrepancy between theory and experiment, that it is not the substrate's non-ideality that is responsible for it.

(iv) General context. The model should consider dynamic wetting not as a one-off exotic phenomenon that needs ad hoc treatment but as a particular case in a more general class of fluid flows which share the same underlying physics. This also implies that the two sides of the problem, namely the "moving contact-line problem' highlighted by Huh and Scriven [25] and the 'dynamic contact angle problem', should be regarded as integral parts of the same physical phenomenon and not as separate elements that can be combined arbitrarily to produce new models (such combined models are described in Sect. 9 of [41]).

The last feature should be seen as a bonus, a certain seal of quality, as inscribing the model of a particular phenomenon in a more general physical context can requires quite some time, as the history of science demonstrated, for example, in electromagnetism.

\subsection{Physical essence of dynamic wetting}

As in Section 2 above, we begin with the static situation. The static (equilibrium) contact angle is introduced in fluid mechanics by the Young equation (1), which is, as already mentioned, nothing but Newton's second law applied to the contact line as one element that constitutes our system as it is described in the framework of continuum mechanics. Since numerous experiments, including those on the nanoscale $[82,83]$, as well as our analysis of Section 3 indicate that in dynamics $\theta_{d} \neq \theta_{s}$, the balance of forces acting on the moving contact line must be different than that in the static case. The question is what makes it different?

The bulk stress cannot be responsible for this change in the force balance simply because stress, being a distributed force, results in a finite force only when it acts on an area; the force it produces while acting on a line is zero (unless the stress is non-integrably singular, which is obviously unphysical).

A concentrated force (i.e., a force per unit length) added to the Young equation (1) can change the contact angle but, whatever force of this kind one might wish to invent and add to the Young equation (the 'contact-line friction force' is but one example), mathematically this will lead to equation (6) and then to an equation similar to (7), which is of the same type as (2). And we have already shown that any formula of this kind as an input in a model would fail our 'litmus test'.

The only option left is to conclude that in dynamics it is the surface tensions featuring in the Young equation (1) that become dynamic, and the contact angle has to adjust to a different force balance. Then, one has the Young equation for the dynamic case in the form

$$
\sigma_{1, \mathrm{dyn}} \cos \theta_{d}=\sigma_{3, \mathrm{dyn}}-\sigma_{2, \mathrm{dyn}},
$$


where $\sigma_{1, \text { dyn }}, \sigma_{2, \text { dyn }}, \sigma_{3, \text { dyn }}$ are the dynamic surface tensions. Then it becomes necessary to consider the dynamics of interfaces and, as a starting point, determine what kind of process is triggered in dynamic wetting flows that creates out-of-equilibrium interfaces and makes the surface tension dynamic.

As explained in [7], the answer is in the very term 'dynamic wetting'. The term means a process by which a liquid spreading over a dry solid surface makes it 'wet', i.e., the process of formation of a new liquid-solid interface, a freshly wet solid surface. This puts dynamic wetting into the general physical context as a particular case in a class of capillary flows where interfaces form and/or disappear. Then, it becomes necessary to develop a theory of such processes and this theory must be developed in the framework of continuum mechanics since it is only there we have an 'interface' as a modelling element.

Thus, (a) the dynamic contact angle deviating from the static one can only be the result of the balance of dynamic surface tensions, and (b) the surface tensions become dynamic as a result of dynamic wetting being a process of interface formation/disapparance. In this process, the liquid-gas interface disappears into the contact line and the fresh liquid-solid interface emerges from the contact line in an out-ofequilibrium state. This interface relaxes to its eventual equilibrium state as it is driven away from the contact line (in the reference frame moving with the contact line), and its dynamics is part of the overall dynamics of the process. This simple picture allows one to rationalize, even on a qualitative level, and describe mathematically all what is observed experimentally in the field of dynamic wetting.

\subsection{The interface formation model versus requirements (i)-(iv)}

The simplest mathematical theory implementing this idea has been formulated in [102] and the first 15 years of its development and application are summarized in [10]. The mathematical essence of the simplest variant of the interface formation model (IFM) is given in the Appendix.

Consider how the IFM performs with regard to criteria (i)-(iv) listed above.

'Litmus test'. In the interface formation model, the dynamic contact angle is part of the solution, the value negotiated by the dynamic surface tensions at the contact line, so the premise of the 'litmus test' is part of how the model was derived. What is required is to test how the model performs once implemented in a numerical code. Although numerical implementation of the interface formation model is a challenging task, considerably harder than implementation of simple slip models, recently this has been done [103,104], thus bringing the use of IFM down to a technical level requiring, at most, the numerical skills of a graduate student.

Figure 7 shows the contact angle-versus-speed curve taken from [103] corresponding to the capillary rise flow. As one can see, the curve has qualitatively exactly the same shape as the curve in Figure 5, including the downward trend where the contact angle decreases as the contact line accelerates. The dashed line in Figure 7 shows the contact angle-versus-speed diagram for the steady contact-line motion (forced spreading). It is noteworthy that this curve differs from the one corresponding to the capillary rise even where the contact angle is close to its equilibrium value, thus confirming that every dynamic wetting process has indeed its individual 'signature' in the contact angle-versus-speed plane.

Qualitative features. The 'rolling' motion of the fluid while spreading on a solid means that the 'fluid particles' which initially belong to the liquid-gas interface reach the contact line in a finite time and then become part of the freshly formed liquid-solid interface. The opposite of this flow kinematics is the so-called 'sliding' motion where the fluid particles belonging to the liquid-gas free surface never reach the contact line which then has to consist of the same fluid particles at all time [1]. 


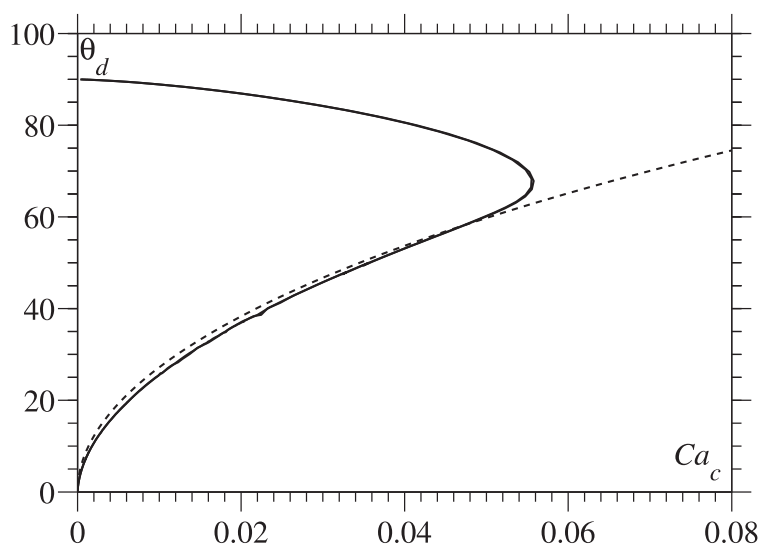

Fig. 7. The contact angle-versus-speed diagram for the capillary rise flow obtained using the interface formation model (solid line) and the corresponding diagram for the steady contact line motion (Fig. 14 from [103]). $C a_{c}=\mu U / \sigma_{1 \text {,eq }}$ is the capillary number (dimensionless contact-line speed), $\theta_{d}$ is the actual contact angle obtained as part of the solution. As one can see, the obtained curve is similar to the one shown in Figure 5 as the result of a qualitative analysis of the flow.

The rolling kinematics has been observed experimentally in different ways $[27,105-$ 107], and it is this very kinematics that is at the core of the interface formation model where one of the boundary conditions at the contact line states that the flux into the contact line due to the liquid-gas interface going into it and disappearing there is equal to the flux out of the contact line along the liquid-solid interface as the latter forms (see the Appendix).

As for 'hydrodynamic assist of dynamic wetting', it should be noted that this effect can be seen as but a particular manifestation of the behaviour of the dynamic contact angle in spontaneous spreading qualitatively analyzed in Section 3 above. Indeed, the contact angle-versus-speed curves of Figures 3-6 show different contact angles corresponding to the same contact-line speed but different accelerations of the contact line as well as the fluid near it and different flow geometries. The latter factor is what the effect of hydrodynamic assist is all about. In this sense, hydrodynamic assist of dynamic wetting as a test of theories should be regarded as a precursor and a particular case of the 'litmus test' considered in Section 3. However, this common physical basis of 'hydrodynamic assist' and the unique angle-versus-speed signature of spontaneous spreading flows is realized only now, though an insightful remark to this effect can be found in [108]. Therefore, the effect of 'hydrodynamic assist' has been studied on its own in the framework of the interface formation model $[109,110]$ and it was shown that this effect is indeed one of the properties of the model.

Quantitative comparison with experiments. Standard flow configurations used in experiments have been outlined in $[1,10]$ and they involve steady and quasisteady capillary flows, so that essentially for a given liquid/fluid/solid system their results come down to a single curve in the contact angle-versus-speed plane. For the interface formation model such a curve is shown as the dashed line in Figure 7. Over the years, the model has been compared and shown to be in good agreement with the results of all standard experiments published in the literature, see [10] for details.

General context. The very name of the interface formation model as the model addressing dynamic wetting inscribes the latter into a general physical context as a particular case in a broad class of flows. In the past 20 years, the model has been applied to some flows from this class, including the following ones: 
- Coalescence of drops, where it removes the singularity at the onset of the process inherent in the conventional model $[111,112]$;

- Breakup of liquid threads, where again it removes the singularity inherent in the conventional model $[113,114]$;

- Rupture of free liquid films [115,116];

- Convergent flows near free surfaces leading to the formation of free-surface cusps/corners discovered experimentally by Joseph et al. [117]. For this type of flow, the conventional model [118] predicts (a) qualitatively wrong kinematics (similar to the sliding motion of the contact line) and (b) the free-surface curvature on the subatomic scale whilst the interface formation model gives a regular solution and remains well within its limits of applicability of continuum mechanics [119].

Further applications included dynamic wetting by a drop impacting on a homogeneous substrate [120] and on a substrate with varying wettability [121], metastable regimes of dynamic wetting [122], flow-induced Marangoni effect [123], and, rather unexpectedly, viscous flows over chemically patterned surfaces $[124,125]$ where no free surfaces are involved. There are now even works studying the interface formation model itself from a purely mathematical point of view addressing the issue of classical solvability of problems formulated in its framework $[126,127]$. In short, the interface formation model has been well inscribed into the general physical context and proved useful in tackling singularities otherwise occurring in the mathematical description of a number of flows where interface formation plays a key role.

Thus, we may conclude that, as far as ideal solid substrates are concerned, the moving contact-line problem has been resolved. The 'litmus test' leaves only the interface formation model to be considered and this model is shown to describe all the relevant features of dynamic wetting and puts this flow in a general physical context.

At the same time, dynamic wetting as a generic physical phenomenon is far from being exhausted, and below we outline some of its aspects that are coming to the forefront and require consolidated research effort.

\section{New challenges}

The resolution of the classical moving contact-line problem described above is largely due to the fact that in the case of an 'ideal' (smooth, chemically homogeneous, rigid, inert) solid substrate we have a uniquely defined static (equilibrium) contact angle and, importantly, the dynamic contact angle is controlled by the same factors (i.e., surface tensions) that determine the static one. Then, it is sufficient to identify the physics that makes these factors dynamic, which is the physics of interface formation, and describe it mathematically to resolve the problem. However, in the processes involving moving contact lines that are now coming to the forefront of research we no longer have these advantages.

\subsection{Rough, chemically inhomogeneous and porous substrates}

If the solid substrate is not ideal, e.g. if it is rough and/or chemically inhomogeneous, which most real substrates are, we no longer have a unique static (equilibrium) contact angle $\theta_{s}$. Instead, one has a static advancing and a static receding contact angle, $\theta_{A}$ and $\theta_{R}$, respectively, which confine the contact angle hysteresis range [60]. For any 
angle between $\theta_{A}$ and $\theta_{R}$ the contact line will be at rest. However, unlike the Young equation (1) which makes $\theta_{s}$ an outcome of the physical factors (surface tensions) whose balance results in the contact line being at rest, we do not have anything of the kind for $\theta_{A}$ and $\theta_{R}$ where additional influences, including even the duration of measurement, are at play [61]. The often cited theories of Wenzel [128,129] and Cassie \& Baxter [130] cannot be regarded as a reliable starting point since not only they have been abundantly disproved experimentally [131-133]; more importantly, they are fundamentally misleading as they relate the contact angle to the surface energy far away from the contact line which is demonstrably not the case [6]. This is important when the goal is not to determine the 'most stable' equilibrium contact angle where these theories provide indicative values [134] but the contact-line motion where the effects of the overall dynamics are dominant.

The contact angle hysteresis is actually a readily observable manifestation of a much deeper issue as the very terms 'rough' and 'chemically inhomogeneous' imply a modelling issue. From a modelling viewpoint, there is a fundamental difference between 'rough' and 'topographically profiled' substrates, 'chemically inhomogeneous' substrates and 'substrates with varying wettability'. The difference lies in the length scale on which the flow is considered. If the length scale $L$ on which the flow is considered is comparable with the length scale $a$ characterizing variation of the substrate's profile, i.e., $L \sim a$, one simply has the contact line moving across a topography; the flow can become three-dimensional but locally, on the size of the topography elements, the substrate is ideal. Similarly, if the characteristic size $a$ of chemical inhomogeneities is comparable with the length scale $L$ on which the flow is described, $a \sim L$, we simply have dynamic wetting of a solid substrate with varying wettability. This is a slight variation on the ideal substrate, and it can be naturally described in the framework of the interface formation model [121].

The substrate becomes 'rough' and/or 'chemically inhomogeneous' if the scales $a$ and $L$ are separated, i.e., in the secondary continuum limit $a / L \rightarrow 0$ (Fig. 8). Essentially, this is the same limit as in mechanics of multiphase media where the secondary continuum limit makes it possible to model phases as occupying the same space overlapping continua and accumulate their properties and interactions into 'effective' (averaged) characteristics, with the effective viscosity of diluted suspensions being perhaps the best known example. The contact angle on a rough and/or chemically inhomogeneous substrate is one of such averaged properties one now has in the description of wetting as now it refers to a length scale large compared with $a$. This is similar to how the actual contact angle on an ideal substrate appears once the length scale $L$ on which the fluid is described becomes large compared with the molecular scale $l_{\mathrm{mol}}$, i.e., in the primary continuum limit $l_{\mathrm{mol}} / L \rightarrow 0$.

From a theoretical point of view, the modelling of wetting phenomena on rough and/or chemically inhomogeneous surfaces is challenging in several ways. As is the case in mechanics of multiphase media, it is not known a priori what concepts must be used to accumulate averaged properties of the actual dynamics of the process. This applies, first of all, to the contact angle and its hysteresis. There is a growing body of experimental data on dynamic wetting of rough substrates [135] but, from a theoretical standpoint, the process is not understood even on a conceptual level.

One of the elements of difficulty is that, for typical rough and/or chemically inhomogeneous surfaces, the size of the roughness elements and/or elements of chemical inhomogeneity is comparable with the slip length on the actual surface of the substrate, so that in the secondary continuum limit slip vanishes into the 'effective contact line' (Fig. 8). Roughness of the substrate per se leads to the no-slip condition for the averaged flow on the effective smooth boundary even with perfect slip on the actual solid surface [136]. Furthermore, both experiments [137] and theory [138-142] have shown that even on a porous surface, which can be regarded as an extreme case of a rough surface, slip is proportional to the pore sizes and hence vanishes in the 


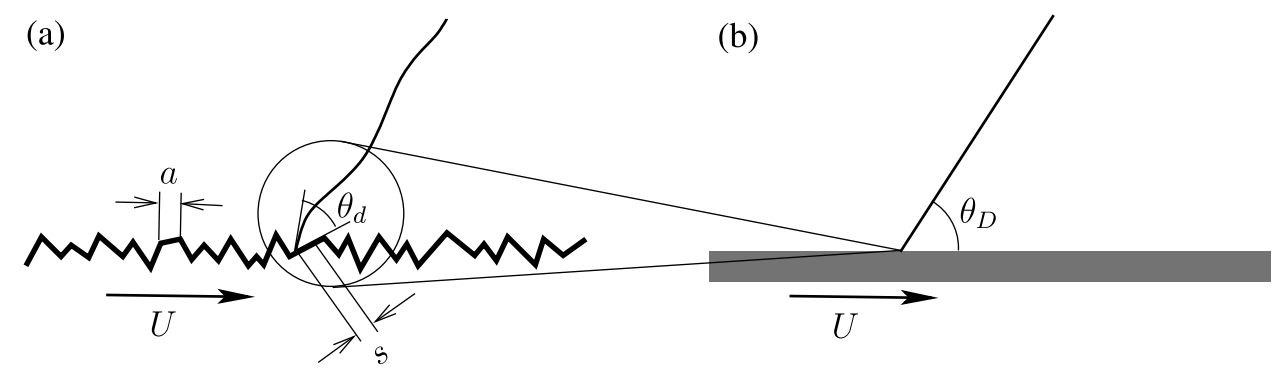

Fig. 8. A sketch illustrating dynamic wetting on a rough substrate. (a) flow over the actual substrate, $\theta_{d}$ is the actual (microscopic) contact angle, $s$ is the slip length, $a$ is the characteristic size of the roughness elements; (b) flow over an 'effective' smooth boundary after the secondary continuum limit $a / L \rightarrow 0$ is taken, $\theta_{D}$ is the 'effective' contact angle. The encircled region is what after the secondary continuum limit becomes the macroscopic 'contact line'.

secondary continuum limit. This leaves one with the no-slip boundary condition on the effective smooth solid boundary, thus excluding slip as a possible remedy for the moving contact-line problem.

Recent experiments [143] also show that, for a rough substrate, the ability of the 'effective' contact line to move strongly depends on connectivity of the actual contact line.

If the solid substrate over which the liquid is spreading is porous, one again has to take the secondary continuum limit as the pore-to-macroscopic size ratio goes to zero and faces essentially the same difficulties as in the case of a rough substrate. However, in the situation where the porous substrate is unsaturated and the material of the porous matrix is wettable by the liquid, the problem appears to be resolvable [144].

The resolution of this problem is instructive. In this case, there is no effective static contact angle as the equilibrium state corresponds to the liquid completely sucked into the porous medium. Then, there is no issue with contact angle hysteresis unavoidable in other situations, but one still has to deal with effective properties. In the absence of the static contact angle, we also do not have explicit factors, like the surface tensions, to specify the effective dynamic contact angle for the spreading liquid. This angle appears as a consequence of the requirement that the solution exists, i.e., as a result of the regularity condition. This requirement links the flow over the substrate with the wetting front propagation in the porous medium and determines the behaviour of the effective dynamic contact angle. This, rather exceptional case, shows that in the situations where the secondary continuum limit has to be taken, the problem resolution often lies on the macroscopic level and is not connected with the mechanism of relieving the moving contact-line singularity on the actual solid substrate.

\subsection{Deformable substrates}

The disparity of dynamic properties of liquids and most solids makes it unnecessary to consider deformation of the solid substrate in many applications. The role of the substrate beyond providing a geometric constraint and, implicitly, the surface tension featuring in the Young equation until recently received relatively little attention albeit from prominent researchers [145-149] who used a variety of approaches and mainly highlighted the issues arising when deformation of the solid substrate is to be taken into account. However, the reversal of dynamic properties of the liquids and solids in an increasing number of applications [150] and, especially, the increasing importance of modelling biological systems with their ubiquitous membranes and soft tissues [151] are now placing elasticity of the solid substrate in static and dynamic wetting 
on the research agenda, primarily experimental [152,153]; see also a recent review [73] for a list of references. In this area, experiments have almost invariably used only one setup, namely a liquid drop placed on a deformable substrate, so that fishing out information about wetting from the overall picture of the drop's statics and dynamics is not always possible. So far, the main research effort has been focussed on the static situation, where experiments have shown that, predictably, the surface tension of the liquid-gas interface pulls and stretches the deformable solid substrate in the direction normal to its unperturbed surface [154] with some indications that the substrate's profile in the immediate neighborhood of the contact line might have a subregion with higher stretching than further afield [155]. The contact angle formed by the liquid-gas free surface with the solid is difficult to measure so that experimental studies typically report an 'effective' angle that the free surface would form with an unperturbed substrate. Experiments on dynamic wetting deal almost exclusively with various 'scaling laws' for the spreading of drops with the common feature being slower motion of the contact line than in the situation where the substrate is rigid $[156,157]$.

On the theoretical side, there is no clarity even with the static situation which is considered mainly in terms of minimization of the free energy with an occasional admixing of disjoining pressure. This approach invariably involves a number of adjustable parameters which allow one to fit the data limited just to the drop-on-asubstrate situation. At the same time, as one can conclude from the case of an ideal substrate, it leads nowhere when it comes to the modelling of dynamic wetting. From the physical viewpoint, the contact-line motion over a deformable substrate is again the process of interface formation where now the model should bring into consideration the dynamics of the solid as well as the fluid and is expected to cover the whole range, from a rigid to a soft gel-like substrate. At present, there is no clarity even on the conceptual level. In particular, with the solid substrate now having been fully involved in the system's dynamics, one might need to revisit the issue of interfacial versus surface tension raised by Gibbs a century ago [52].

\subsection{Dynamic wetting and phase transitions}

Fluid flows where dynamic wetting occurs in parallel with one or more of phase transitions (evaporation, condensation, solidification, melting) are becoming an increasingly important topic of research due to many applications, actual and/or potential, in a number of technologies (e.g. 3D printing) and, to some extent, as relevant to the safety issues (e.g. icing of aircraft).

Conceptually, these flows are much simpler for modelling than dynamic wetting of non-ideal substrates where one has to consider the secondary continuum limit and deal with averaged quantities, though in the phase transition case, like in the case of unsaturated wettable porous substrates, there is no static (equilibrium) contact angle. The key point to note here is that phase transitions are processes additional to dynamic wetting. Once phase transitions are taken out, the mathematical model intended to describe dynamic wetting with phase transitions should reduce to an adequate model of dynamic wetting itself, i.e., to a model satisfying the requirements itemized as (i)-(iv) in Section 4. If this hydrodynamic limit recovers just a formula for the dynamic contact angle as a function of the contact-line speed, e.g. Voinov's [78] or Cox's [80] expressions, (9) and (11) respectively, which, as we show in Section 3, fail to describe numerous wetting flows, there is no hope that a theory built on top of these models to account for phase transitions, e.g. solidification, would give an adequate description of the corresponding flow.

Experiments on dynamic wetting of undercooled substrates [158-161] show that the process has two distinct stages. A drop deposited onto a cold substrate spreads as 
if in an isothermal environment but very shortly the contact-line motion is arrested, and the remaining process is that of freezing. Experiments are typically accompanied by proto-theories $[159,160,162]$ with the current understanding attributing the arrest of the contact-line motion to kinetic undercooling [160]. Ironically, the fluid mechanical side of the process is handled on the basis of the formulae making the dynamic contact angle a function of the contact-line speed, which we analyzed in Section 2 above, and the no-slip boundary condition, whilst descriptively experimentalists refer to "the usual spreading, where fresh liquid is continuously supplied to the contact line" [160], which is only one step away from the idea of interface formation and the corresponding model. A recent work by Thiévenaz et al. [163] links pinning of the contact line to the one-dimensional growth of an ice sheet developing between the substrate and the liquid, but an adequate theory of the whole process is still lacking.

Dynamic wetting in the situation, where, instead of the liquid solidifying, the substrate liquifies, has been studied mostly in the context of the so-called reactive or dissolutive wetting $[164,165]$ with applications primarily in metallurgy. The model describing this phenomenon must again cover the whole range of regimes, from the contact line pinned to the rim formed by dissolving and intact parts of the substrate and moving as the substrate dissolves and the rim propagates to the hydrodynamic limit, where no substrate dissolution takes place and one has dynamic wetting in its classic sense. The latter can then be used as a preliminary check of the adequacy of the model with our 'litmus test' at hand to help. Theories proposed to describe the process, e.g. [166], are shown in the hydrodynamic limit to reduce to the Cox-Voinov formulae [167]. This indicates that such models are inadequate at least close to the hydrodynamic limit, i.e., for weakly dissolving substrates. A review of dissolutive wetting can be found in [168].

Dynamic wetting with evaporation-condensation occurring in the vicinity of the moving contact line is the case where the phase transition takes place on the free surface. Currently, the main effort in this area is directed towards rather exotic situations, e.g. a possible removal of the contact-line singularity via evaporation-condensation $[169,170]$ or thermal effects being the driver of the contact-line motion [171], i.e., the situations where, should the phase transition be taken out, no dynamic wetting takes place. This obviously leaves the problem of modelling dynamic wetting with evaporation-condensation processes as additional to dynamic wetting open.

An important point to be noted about this type of flows is that in the continuum mechanics modelling the contact line is not just a line with which the liquid-vapour interface ends; it is an element of the system in its own right, with other elements being the 'bulk phases' and sharp 'interfaces'. In other words, evaporation of the contact line (i.e., physically, the three-phase-interaction zone) is to be considered conceptually separately from the evaporation from the free surface. An example of a separate role of the contact line is given in the interface formation model (see the Appendix), where the role played by the dynamic properties of the contact line is negligible (it always is, see an emphatic piece in de Gennes et al. [8] regarding the 'myth of the line tension') but it acts as a 'connector' of the properties of the liquidgas and liquid-solid interface. Adding evaporation-condensation to the model does not look like a conceptually difficult task but this might turn out to be not so simple as it seems.

\section{Concluding remarks: mathematics, physics and modelling}

As reviewed in Section 2, the search for the formula describing the velocitydependence of the dynamic contact angle has been on for 50 years, from Blake and Haynes paper of 1969 [67] all the way to the present day, and yet an elementary qualitative analysis of some basic spontaneous spreading flows given in Section 3 
shows that all this research effort for half a century has been in essence a wild goose chase: the coveted formula simply does not exist.

How could it happen that the entire dynamic wetting community almost without exceptions was chasing this idiomatic bird for so long? This is not an idle question as without analyzing the reasons behind what happened and learning from this analysis, there is no guarantee that this will not happen again, say, in addressing one of the new challenges emerging in the field some of which are outlined in Section 5. One consideration that might contribute to this analysis is as follows.

Being at the intersection of many applications and several research disciplines, dynamic wetting draws in researchers with different educational backgrounds but ultimately the knowledge about the process is expected to be cast in terms and concepts of continuum mechanics. It is the language of continuum mechanics that is used by experimentalists, and the results obtained by other methods, e.g. molecular dynamics and lattice Boltzmann simulations, have to be converted into this language. In this context, continuum mechanics is no longer a mathematical art of finding particular/approximate solutions of the classical systems of nonlinear partial differential equations with known boundary conditions, which it has been for almost two centuries; it is a modelling approach with its own conceptual and mathematical framework. All 'subcontinuum' molecular-level effects have to be wrapped up into its macroscopic concepts in a certain way, not just added on top of existing models.

On the other hand, the outcome of the modelling is expected to satisfy the same standards of mathematical rigour as in the subject's purely mathematical past and for every particular flow one should have a closed well-posed mathematical problem which could then be solved formally, without any 'cut-offs', 'mesoscopic' arguments and other verbal substitutes for mathematics. This duality of modern continuum mechanics and the kind of specialization it requires seems to be at the core of what happened in dynamics wetting.

Applied mathematicians, like Voinov [78] or Cox [80], trained in fluid mechanics as a branch of mathematics, treated the problem as mathematical within a given model and came up with their solutions. The adequacy of the model itself was neither questioned nor even considered. However, what the dynamic wetting community needed was a model, not a solution to a particular problem in the framework of a model with question marks hanging over it. As a result of this conflict between supply and demand, the velocity-dependence of the 'apparent' contact angle derived by Voinov and Cox for a particular flow in the framework of particular models, as well as an even simpler expression obtained by Tanner [87] for another particular flow, became detached from the problems these authors studied and started being used either as elements of newly proposed models or even as benchmarks against which such models now have been tested. Thus, the schooling in continuum mechanics as a branch of applied mathematics is of little help when it comes to mathematical modelling, and the mathematically correct results can be misused by other modelers, as it happened with Voinov's, Cox's and Tanner's work. Venturing into mathematical modelling without schooling in it albeit with a mathematical background would be ill-advised as this often leads to confusion about elementary things [57,58].

One might expect that those trained in continuum mechanics on a qualitative physical level and at home with the physical essence of new phenomena might have been better equipped to deal with the problems arising in dynamic wetting but this turned out not to be the case. Physicists, chemists and engineers typically lack the required mathematical background, and their 'mathematical models', if they happen to be formulated in the framework of continuum mechanics, often include 'molecular cut-offs', 'mesoscopic' justification of inconsistencies and other forms of verbal support, not to mention the lubrication approximation of non-existent solutions. Insightful reviews of physics [2] and books in the style of 'scientific impressionism' [8], enjoyable as they are, leave one wondering what to do with all these words and 
equations brought in just to illustrate them should one wish to actually make mathematics work, describe a particular process involving dynamic wetting as the solution of a closed well-posed mathematical problem? Tellingly, multiple publications following from the works of de Gennes and co-authors [172,173] limit their scope to perfect or near-perfect wetting, thus avoiding the need to face the dynamic contact angle problem as well as fluid mechanics as such beyond the lubrication approximation. So, non-mathematicians are of little help with the modelling either as their qualitative understanding of the relevant physics is yet to be channeled into the kind of mathematics that could stand scrutiny.

Thus, one of the main reasons behind the misguided 50-year-long search for the non-existent formula relating the dynamic contact angle with the contact-line speed seems to be that the nature of continuum mechanics has changed in the past few decades, it became a nontrivial modelling framework but still with rigorous mathematical standards, whilst the education and expertise of those who come into it, especially in multidisciplinary areas of research, like dynamic wetting, are lagging behind this change. As a matter of fact, only in a handful of universities worldwide one can find continuum mechanics taught as a central course alongside mathematics and physics; even fewer teach it as a modelling framework and fewer still give their graduates an opportunity to consolidate and further develop the acquired modelling skills in a research environment. As for scientific schools, the schools of mathematical modelling in general and of the modelling with an emphasis on continuum mechanics as a modelling approach in particular, they are not simply rare, their very existence is under threat from how science is now being organized and funded.

At the same time, the complexity of the physical phenomena needed to be modelled in the framework of continuum mechanics and the complexity of the corresponding models are increasing as the latter are no longer constrained by what analytic methods can handle. In this trend, dynamic wetting is but one example. All this calls for researchers with training and experience in both mathematics and mathematical modelling as a special skill. Self-education helps but it is not a reliable substitute for regular schooling when it comes to new physics and new models. To put it in simple terms: no matter how much one trawls the literature and how often one comes across the notion of the 'right angle', one will need schooling not to start searching for the left one. Or, equivalently, for a function relating the contact angle with the contact-line speed.

The support of the Engineering and Physical Sciences Research Council (U.K.) under Grant $\mathrm{EP} / \mathrm{P} 031684 / 1$ is acknowledged.

Open Access This is an open access article distributed under the terms of the Creative Commons Attribution License (http://creativecommons.org/licenses/by/4.0), which permits unrestricted use, distribution, and reproduction in any medium, provided the original work is properly cited.

Publisher's Note The EPJ Publishers remain neutral with regard to jurisdictional claims in published maps and institutional affiliations.

\section{Appendix: Mathematical summary of the interface formation model}

For the case of a viscous incompressible Newtonian fluid of density $\rho$ and viscosity $\mu$ spreading over a smooth chemically homogeneous non-reactive rigid solid surface and surrounded by an inviscid dynamically passive gas at a constant pressure $p_{g}$, the 
simplest (irreducible) interface formation model takes the following form [10]. The fluid's velocity $\mathbf{u}$ and pressure $p$ satisfy the Navier-Stokes equations,

$$
\nabla \cdot \mathbf{u}=0, \quad \rho\left(\frac{\partial \mathbf{u}}{\partial t}+\mathbf{u} \cdot \nabla \mathbf{u}\right)=-\nabla p+\mu \nabla^{2} \mathbf{u}
$$

subject to the following boundary conditions. On the free surface defined as $f(\mathbf{r}, t)=0$, where the function $f$ is to be found, one has

$$
\begin{gathered}
\frac{\partial f}{\partial t}+\mathbf{v}_{1}^{s} \cdot \nabla f=0 \\
p_{g}-p+\mu \mathbf{n}_{1} \cdot\left[\nabla \mathbf{u}+(\nabla \mathbf{u})^{T}\right] \cdot \mathbf{n}_{1}=\sigma_{1} \nabla \cdot \mathbf{n}_{1}, \\
\mu \mathbf{n}_{1} \cdot\left[\nabla \mathbf{u}+(\nabla \mathbf{u})^{T}\right] \cdot\left(\mathbf{I}-\mathbf{n}_{1} \mathbf{n}_{1}\right)+\nabla \sigma_{1}=0, \\
\rho\left(\mathbf{u}-\mathbf{v}_{1}^{s}\right) \cdot \mathbf{n}_{1}=\frac{\rho_{1}^{s}-\rho_{1, \mathrm{eq}}^{s}}{\tau}, \\
\frac{\partial \rho_{1}^{s}}{\partial t}+\nabla \cdot\left(\rho_{1}^{s} \mathbf{v}_{1}^{s}\right)=-\frac{\rho_{1}^{s}-\rho_{1, \mathrm{eq}}^{s}}{\tau}, \\
(1+4 \alpha \beta) \nabla \sigma_{1}=4 \beta\left(\mathbf{v}^{s}-\mathbf{u}\right) \cdot\left(\mathbf{I}-\mathbf{n}_{1} \mathbf{n}_{1}\right),
\end{gathered}
$$

whilst on the liquid-solid interface the boundary conditions take the form

$$
\begin{gathered}
\left(\mathbf{v}_{2}^{s}-\mathbf{U}\right) \cdot \mathbf{n}_{2}=0 \\
\mu \mathbf{n}_{2} \cdot\left[\nabla \mathbf{u}+(\nabla \mathbf{u})^{T}\right] \cdot\left(\mathbf{I}-\mathbf{n}_{2} \mathbf{n}_{2}\right)+\frac{1}{2} \nabla \sigma_{2}=\beta(\mathbf{u}-\mathbf{U}) \cdot\left(\mathbf{I}-\mathbf{n}_{2} \mathbf{n}_{2}\right), \\
\rho\left(\mathbf{u}-\mathbf{v}_{2}^{s}\right) \cdot \mathbf{n}_{2}=\frac{\rho_{2}^{s}-\rho_{2, \mathrm{eq}}^{s}}{\tau}, \\
\frac{\partial \rho_{2}^{s}}{\partial t}+\nabla \cdot\left(\rho_{2}^{s} \mathbf{v}_{2}^{s}\right)=-\frac{\rho_{2}^{s}-\rho_{2, \mathrm{eq}}^{s}}{\tau} \\
{\left[\mathbf{v}_{2}^{s}-\frac{1}{2}(\mathbf{u}+\mathbf{U})-\alpha \nabla \sigma_{2}\right] \cdot\left(\mathbf{I}-\mathbf{n}_{2} \mathbf{n}_{2}\right)=0 .}
\end{gathered}
$$

Here subscripts 1 and 2 mark the surface parameters of the liquid-gas and liquidsolid interface, respectively; $\sigma, \rho^{s}$ are the surface tension and the surface density characterizing the interfaces, $\mathbf{v}^{s}$ is the velocity whose tangential to the interface component describes flow in the interface as a two-dimensional continuum and the normal component describes the motion of the interface itself; $\mathbf{n}$ is a unit normal to the interface pointing into the fluid and $\mathbf{I}$ is the metric tensor, so that the tensor 
$(\mathbf{I}-\mathbf{n n})$ extracts the vector component parallel to the interface, e.g. $\mathbf{u} \cdot(\mathbf{I}-\mathbf{n n})=$ $\left(\mathbf{u}_{\|}+u_{n} \mathbf{n}\right) \cdot(\mathbf{I}-\mathbf{n n})=\mathbf{u}_{\|} ; \mathbf{U}$ is the velocity of the solid substrate; $\alpha, \beta, \tau, \rho_{1, \text { eq }}^{s}, \rho_{2, \text { eq }}^{s}$ are constants characterizing transport within interfaces $(\alpha, \beta)$, equilibrium surface densities $\left(\rho_{1, \text { eq }}^{s}, \rho_{2, \text { eq }}^{s}\right)$ and the interface relaxation time $(\tau)$.

Equations (A.2)-(A.4) are the standard kinematic, normal- and tangential-stress conditions $\left(\nabla \cdot \mathbf{n}_{1}\right.$ is the mean curvature of the free surface), (A.5) and (A.6) describe the bulk-interface mass exchange and the relaxation of the surface density to its equilibrium value, and (A.7) accounts for the difference between the tangential components of the surface velocity and the bulk velocity evaluated at the interface due to the torque formed by the surface tension gradient acting in the interface and the shear stress acting on its liquid-facing side. Equation (A.9) is the generalized Navier condition accounting for slip due to shear stress and the surface tension gradient; note that $\mathbf{u}$ in this equation is the bulk velocity evaluated on the liquid-facing side of the liquid-solid interface whilst on the solid-facing side of it there is no slip between the interface and the solid surface. Equation (A.10), (A.11) are similar to (A.5), (A.6) and describe the bulk-interface mass exchange and the relaxation of $\rho_{2}^{s}$ to its equilibrium value $\rho_{2, \text { eq }}^{s}$; (A.12) characterizes transport within the liquid-solid interface (it has the same form as for the average velocity in a channel described by the combined Couetta-Poiseuille solution).

The sets of boundary conditions (A.2)-(A.7) and (A.8)-(A.12) must be closed by the surface equation of state relating, in the simplest case, the surface tension $\sigma$ and the surface density $\rho^{s}$. This equation can be taken in a linear form

$$
\sigma_{i}=\gamma\left(\rho_{0}^{s}-\rho_{i}^{s}\right), \quad(i=1,2)
$$

reflecting the fact that rarefaction of the interface increases its surface tension; $\gamma$ and $\rho_{0}^{s}$ are constants.

Finally, at the moving contact line, where the liquid-gas and the liquid-solid interfaces meet, one needs conditions linking the distributions of the surface parameters along these interfaces. In the coordinate frame moving with the contact line, the conditions have the form

$$
\begin{gathered}
\left(\rho_{1}^{s} \mathbf{v}_{1}^{s}\right) \cdot \mathbf{e}_{1}+\left(\rho_{2}^{s} \mathbf{v}_{2}^{s}\right) \cdot \mathbf{e}_{2}=0 \\
\sigma_{1}\left(\rho_{1}^{s}\right) \cos \theta_{d}+\sigma_{2}\left(\rho_{2}^{s}\right)=0
\end{gathered}
$$

where $\mathbf{e}_{1}, \mathbf{e}_{2}$ are unit vectors normal to the contact line and pointing into the liquidgas and the liquid-solid interface, respectively; $\theta_{d}$ is the dynamic contact angle defined as $\theta_{d}=\arccos \left(-\mathbf{n}_{1} \cdot \mathbf{n}_{2}\right) \equiv \arccos \left(\mathbf{e}_{1} \cdot \mathbf{e}_{2}\right)$. Equation (A.14) is the balance of mass fluxes in and out of the contact line, and (A.15) is the Young equation for dynamic surface tensions expressing the balance of the forces acting on the contact line in the direction tangential to the substrate. In equilibrium, one recovers the classical Young equation

$$
\sigma_{1}\left(\rho_{1, \mathrm{eq}}^{s}\right) \cos \theta_{s}+\sigma_{2}\left(\rho_{2, \mathrm{eq}}^{s}\right)=0
$$

where $\theta_{s}$ is the static (equilibrium) contact angle. As we remember, in a liquid/gas/solid system the surface tension of the gas-solid interface in (1) is zero. Note that the contact line has no dynamic properties (like the 'line tension') but serves as a 'connector' linking the distributions of the surface parameters along the interfaces.

Equations (A.1)-(A.15) completed with the appropriate initial conditions and conditions specifying a particular flow allow one to describe it mathematically, simply 
as the solution of a mathematical problem without any non-mathematical 'cut-offs' nor any other forms of verbal support.

It should be noted that the model (A.1)-(A.15) is formulated consistently in the framework of classical fluid mechanics; the interfaces are sharp, without 'interfacial thickness' or 'diffuse interface' featuring anywhere, and does not include any intermolecular forces on top of its macroscopic quantities. All parameters involved have clear physical meanings (see [10] for details) and can be estimated or determined from experiments [174].

The model has been generalized to the case of a liquid/liquid/solid system [10] and provides a modelling framework allowing one to incorporate other factors and physical effects (surfactants, thermal effects, chemical reactions, etc) in a regular way.

\section{References}

1. E.B. Dussan, Annu. Rev. Fluid Mech. 11, 371 (1979)

2. P.-G. de Gennes, Rev. Mod. Phys. 57, 827 (1985)

3. Y. Pomeau, C. R. Mécanique 330, 207 (2002)

4. T.D. Blake, J. Colloid Interface Sci. 299, 1 (2006)

5. J. Ralston, M. Popescu, R. Sedev, Annu. Rev. Mater. Res. 38, 23 (2008)

6. L. Gao, T.J. McCarthy, Langmuir 25, 14105 (2009)

7. Y.D. Shikhmurzaev, Eur. Phys. J. Special Topics 197, 47 (2011)

8. P.-G. de Gennes, F. Brochard-Wyat, D. Quéré, Capillarity and Wetting Phenomena: Drops, Bubbles, Pearls, Waves (Springer, 2004)

9. V.M. Starov, M.G. Velarde, C.J. Radke, Wetting and Spreading Dynamics (CRC Press, 2007)

10. Y.D. Shikhmurzaev, Capillary Flows with Forming Interfaces (Chapman \& Hall/CRC, Boca Raton-London-New York, 2007)

11. U. Thiele, Eur. Phys. J. Special Topics 197, 67 (2011)

12. Y.D. Shikhmurzaev, Eur. Phys. J. Special Topics 197, 221 (2011)

13. L.M. Pismen, Eur. Phys. J. Special Topics 197, 63 (2011)

14. Y.D. Shikhmurzaev, Eur. Phys. J. Special Topics 197, 75 (2011)

15. Y. Pomeau, Eur. Phys. J. Special Topics 197, 81 (2011)

16. Y.D. Shikhmurzaev, Eur. Phys. J. Special Topics 197, 85 (2011)

17. J.R. Henderson, Eur. Phys. J. Special Topics 197, 61 (2011)

18. Y.D. Shikhmurzaev, Eur. Phys. J. Special Topics 197, 125 (2011)

19. T.D. Blake, Eur. Phys. J. Special Topics 197, 343 (2011)

20. Y.D. Shikhmurzaev, Eur. Phys. J. Special Topics 197, 73 (2011)

21. G.D. West, Proc. Roy. Soc. A86, 20 (1911)

22. W.B. Hardy, Philos. Mag. 38, 49 (1919)

23. R. von Lucas, Koll. Zeitschr. 23, 15 (1918)

24. E.W. Washburn, Phys. Rev. 17, 273 (1921)

25. C. Huh, L.E. Scriven, J. Colloid Interface Sci. 35, 85 (1971)

26. H.K. Moffatt, J. Fluid Mech. 18, 1 (1964)

27. E.B. Dussan, S.H. Davis, J. Fluid Mech. 65, 71 (1974)

28. E.B. Dussan, J. Fluid Mech. 77, 665 (1976)

29. L.M. Hocking, J. Fluid Mech. 79, 209 (1977)

30. C. Huh, S.G. Mason, J. Fluid Mech. 81, 401 (1977)

31. H.P. Greenspan, J. Fluid Mech. 84, 125 (1978)

32. P.A. Durbin, J. Fluid Mech. 197, 157 (1988)

33. M.Y. Zhou, P. Sheng, Phys. Rev. Lett. 64, 882 (1990)

34. T. Qian, X.-P. Wang, P. Sheng, Phys. Rev. E 68, 016306 (2003)

35. W. Ren, Phys. Fluids 19, 022101 (2007)

36. J.J. Thalakkottor, K. Mohseni, Phys. Rev. E 94, 023113 (2016)

37. K. Yokoi, D. Vadillo, J. Hinch, I. Hutchings, Phys. Fluids 21, 072102 (2009) 
38. S. van Mourik, A.E.P. Veldman, M.E. Dreyer, Microgravity Sci. Technol. 17, 87 (2005)

39. O. Weinstein, L.M. Pismen, Math. Modelling Nat. Phenomena 3, 98 (2008)

40. M. Navier, Mem. Acad. Sci. Inst. France 6, 389 (1823)

41. Y.D. Shikhmurzaev, J. Fluid Mech. 334, 211 (1997)

42. D.N. Sibley, N. Savva, S. Kalliadasis, Phys. Fluids 24, 082105 (2002)

43. P. Colinet, A. Rednikov, Eur. Phys. J. Special Topics 197, 89 (2011)

44. D.J. Benney, W.J. Timson, Stud. Appl. Math. 63, 93 (1980)

45. L.M. Pismen, A. Nir, Phys. Fluids 25, 3 (1982)

46. C.G. Ngan, E.B. Dussan, Phys. Fluids 27, 2785 (1984)

47. J. Koplik, J.R. Banavar, J.F. Willemsen, Phys. Rev. Lett. 60, 1282 (1988)

48. P.A. Thompson, M.O. Robbins, Phys. Rev. Lett. 63, 766 (1989)

49. J.L. Barrat, L. Bocquet, Phys. Rev. Lett. 82, 4671 (1999)

50. H. Brenner, V. Ganesan, Phys. Rev. E 61, 6879 (2000)

51. T. Young, Trans. Roy. Soc. (London) 95, 65 (1805)

52. J.W. Gibbs, in Collected Works of J. Willard Gibbs (Longmans, Green \& Co., New York, 1928), Vol. 1

53. G.J. Merchant, J.B. Keller, Phys. Fluids A 4, 477 (1992)

54. D. Seveno, T.D. Blake, J. De Coninck, Phys. Rev. Lett. 111, 096101 (2013)

55. A.V. Lukyanov, A.E. Likhtman, ACS Nano 10, 6045 (2016)

56. J-C. Fernandez-Toledano, T.D. Blake, J. De Coninck, Langmuir 33, 2929 (2017)

57. R. Finn, Phys. Fluids 18, 047102 (2006)

58. Y.D. Shikhmurzaev, Phys. Lett. A 372, 704 (2008)

59. Lord Rayleigh, Phil. Mag. 30, 386 (1890)

60. H.B. Eral, D.J.C.M. Mannetje, J.M. Oh, Colloid Polym. Sci. 291, 247 (2013)

61. J.W. Drelich, Adv. Colloid \& Interf. Sci. 267, 1 (2019)

62. R. Ablett, Philos. Mag. 46, 244 (1923)

63. G.D. Yarnold, B.J. Mason, Proc. Phys. Soc. (London) B62, 125 (1949)

64. P.J. Haley, M.J. Miksis, J. Fluid Mech. 223, 57 (1991)

65. P. Ehrhard, S.H. Davis, J. Fluid Mech. 229, 365 (1991)

66. Y. Xia, P.H. Steen, J. Fluid Mech. 841, 767 (2018)

67. T.D. Blake, J.M. Haynes, J. Colloid Interface Sci. 30, 421 (1969)

68. T.D. Blake, A. Clarke, J. De Coninck, M.J. de Ruijter, Langmuir 13, 2164 (2013)

69. J-C. Fernandez-Toledano, T.D. Blake, J. De Coninck, J. Colloid Interface Sci. 540, $322(2019)$

70. T. Qian, X.-P. Wang, P. Sheng, J. Fluid Mech. 564, 333 (2006)

71. W. Ren, Phys. Fluids 22, 102103 (2010)

72. P. Yue, J.J. Feng, Phys. Fluids 23, 012106 (2011)

73. L. Chen, E. Bonaccurso, T. Gambaryan-Roisman, V. Starov, N. Koursari, Y. Zhao, Curr. Opin. Colloid Interf. Sci. 36 (2018)

74. P. Johansson, B. Hess, Phys. Rev. Fluids 3, 074201 (2018)

75. X. Xu, Y. Di, H. Yu, J. Fluid Mech. 849, 805 (2018)

76. A. Reusken, X. Xu, L. Zhang, Intl J. Numer. Meth. Fluids 84, 268 (2017)

77. H.S.H. Mohand, H. Hoang, G. Galliero, D. Legendre, J. Comput. Phys. 393, 29 (2019)

78. O.V. Voinov, Sov. Phys. - Doklady 23, 891 (1978)

79. S.H. Davis, J. Fluid Mech. 98, 225 (1980)

80. R.G. Cox, J. Fluid Mech. 168, 169 (1986)

81. E.L. Decker, B. Frank, Y. Suo, S. Garoff, Colloids Surf. A 156, 177 (1999)

82. L. Chen, J. Yu, H. Wang, ACS Nano 8, 11493 (2014)

83. Y. Deng, L. Chen, Q. Liu, J. Yu, H. Wang, J. Phys. Chem. Lett. 7, 1763 (2016)

84. Q. Wu, H. Wong, J. Fluid Mech. 506, 157 (2004)

85. J. Eggers, H.A. Stone, J. Fluid Mech. 505, 309 (2004)

86. P.G. Petrov, J. Petrov, Langmuir 8, 1762 (1992)

87. L.H. Tanner, J. Phys. D: Appl. Phys. 12, 1473 (1979)

88. A. Milchev, K. Binder, J. Chem. Phys. 116, 7691 (2002)

89. G. He, N.G. Hadjiconstantinou, J. Fluid Mech. 497, 123 (2003)

90. L. Giacomelli, M.V. Gnann, F. Otto, Nonlinearity 29, 2497 (2016) 
91. D. Seveno, A. Vaillant, R. Rioboo, H. Adao, J. Conti, J. De Coninck, Langmuir 25, 13034 (2009)

92. M.J. de Ruijter, J. De Coninck, G. Oshanin, Langmuir 15, 2209 (1999)

93. M.J. Davis, S.H. Davis, C. R. Phys. 14, 629 (2013)

94. A.M. Karim, S.H. Davis, H.P. Kavehpour, Langmuir 32, 10153 (2016)

95. T.D. Blake, A. Clarke, K.J. Ruschak, AIChE J. 40, 229 (1994)

96. T.D. Blake, M. Bracke, Y.D. Shikhmurzaev, Phys. Fluids 11, 1995 (1999)

97. A. Clarke, E. Stattersfield, Phys. Fluids 18, 048109 (2006)

98. C.-Y. Liu, E. Vandre, M.S. Carvalho, S. Kumar, J. Fluid Mech. 808, 290 (2016)

99. M.C.T. Wilson, J.L. Summers, Y.D. Shikhmurzaev, A. Clarke, T.D. Blake, Phys. Rev. E 73, 041606 (2006)

100. I.S. Bayer, C.M. Megaridis, J. Fluid Mech. 558, 415 (2006)

101. Y.D. Shikhmurzaev, Physica D 217, 121 (2006)

102. Y.D. Shikhmurzaev, Intl J. Multiphase Flow 19, 589 (1993)

103. J.E. Sprittles, Y.D. Shikhmurzaev, J. Comput. Phys. 233, 34 (2013)

104. J.E. Sprittles, Y.D. Shikhmurzaev, J. Comput. Phys. 274, 936 (2014)

105. A.M. Schwartz, C.A. Rader, E. Huey, in Contact Angle, Wettability and Adhesion, edited by R.F. Gould (ACS, Washington, DC, 1964), pp. 250-267

106. A. Clarke, Chem. Eng. Sci. 50, 2397 (1995)

107. Q. Chen, E. Ramé, S. Garoff, Colloids Surf. 116, 115 (1996)

108. T.D. Blake, J.-C. Fernandez-Toledano, G. Doyen, J. De Coninck, Phys. Fluids 27, $012101(2015)$

109. A.V. Lukyanov, Y.D. Shikhmurzaev, Phys. Lett. A 358, 426 (2006)

110. A.V. Lukyanov, Y.D. Shikhmurzaev, Phys. Rev. E 75, 051604 (2007)

111. J.E. Sprittles, Y.D. Shikhmurzaev, Phys. Fluids 24, 122105 (2012)

112. J.E. Sprittles, Y.D. Shikhmurzaev, J. Fluid Mech. 751, 480 (2014)

113. Y.D. Shikhmurzaev, IMA J. Appl. Math. 70, 880 (2005)

114. Y. Li, J.E. Sprittles, J. Fluid Mech. 797, 29 (2016)

115. Y.D. Shikhmurzaev, C. R. Mecanique 333, 205 (2005)

116. Y.D. Shikhmurzaev, Intl Polym. Process. 22, 38 (2007)

117. D.D. Joseph, J. Nelson, M. Renardy, Y. Renardy, J. Fluid Mech. 223, 383 (1991)

118. J.-T. Jeong, H.K. Moffatt, J. Fluid Mech. 241, 1 (1992)

119. Y.D. Shikhmurzaev, Phys. Lett. A 345, 378 (2005)

120. M. Griebel, M. Klitz, Comput. \& Math. with Appl. 78, 3027 (2019)

121. J.E. Sprittles, Y.D. Shikhmurzaev, Phys. Fluids 24, 082001 (2012)

122. Y.D. Shikhmurzaev, J. Phys.: Condens. Matter 14, 319 (2002)

123. Y.D. Shikhmurzaev, AIChE J. 42, 601 (1997)

124. J.E. Sprittles, Y.D. Shikhmurzaev, Phys. Rev. E 76, 021602 (2007)

125. J.E. Sprittles, Y.D. Shikhmurzaev, Eur. Phys. J. Special Topics 166, 159 (2009)

126. Y. Kusaka, Anal. Math. Phys. 5, 67 (2015)

127. Y. Kusaka, Anal. Math. Phys. 6, 109 (2016)

128. R.N. Wenzel, Ind. Eng. Chem. 28, 988 (1936)

129. R.N. Wenzel, J. Phys. Colloid Chem. 53, 1466 (1949)

130. A.B.D. Cassie, S. Baxter, Trans. Faraday Soc. 40, 546 (1944)

131. V.R. Gray, Chem. Ind. 23, 969 (1965)

132. G. Wolansky, A. Marmur, Colloid Surf. A 156, 381 (1999)

133. L. Gao, T.J. McCarthy, Langmuir 23, 3762 (2007)

134. T.S. Meiron, A. Marmur, I.S. Saguy, J. Colloid Interface Sci. 274, 637 (2004)

135. D. Quéré, Annu. Rev. Mater. Res. 38, 71 (2008)

136. S. Richardson, J. Fluid Mech. 59, 707 (1973)

137. G.S. Beavers, D.D. Joseph, J. Fluid Mech. 30, 197 (1967)

138. P.G. Saffman, Stud. Appl. Maths 50, 93 (1971)

139. I.P. Jones, Proc. Cambridge Philos. Soc. 73, 231 (1973)

140. A.I. Murdoch, A. Soliman, Proc. R. Soc. (London) A 455, 1315 (1999)

141. D.A. Nield, Transp. Porous Media 78, 537 (2009)

142. J.-L. Auriault, Transp. Porous Media 83, 257 (2010) 
143. Y. Jiang, Y. Sun, J.W. Drelich, C.-H. Choi, Langmuir 34, 4945 (2018)

144. Y.D. Shikhmurzaev, J.E. Sprittles, J. Fluid Mech. 715, 273 (2013)

145. G.R. Lester, J. Colloid Sci. 16, 315 (1961)

146. A.I. Rusanov, Colloid J. USSR 37, 614 (1975)

147. B.V. Deryagin, V.M. Starov, N.V. Churaev, Colloid J. USSR 44, 770 (1982)

148. M.E.R. Shanahan, P.-G. de Gennes, C. R. Acad. Paris 2, 517 (1986)

149. M.E.R. Shanahan, J. Phys. D : Appl. Phys. 20, 945 (1987)

150. A. Hirsch, L. Dejace, H.O. Michaud, S.P. Lacour, Acc. Chem. Res. 52, 534 (2019)

151. R.A. Samy, A.K. Sen, J. Micromech. Microeng. 29, 065001 (2019)

152. A. Leh, H.E. N'guessan, J. Fan, P. Bahadur, R. Tadmor, Y. Zhao, Langmuir 28, 5795 (2012)

153. B. Andreotti, J. Snoeijer, Europhys. Lett. 113, 66001 (2016)

154. R. Pericet-Camara, G.R. Auernhammer, K. Koynov, S. Lorenzoni, R. Raiteri, E. Bonaccurso, Soft Matter 5, 3611 (2009)

155. G. Pu, J.H. Guo, L.E. Gwin, S.J. Severtson, Langmuir 23, 12142 (2007)

156. A. Carre, M.E.R. Shanahan, Langmuir 11, 24 (1995)

157. A. Carre, J.C. Gastel, M.E.R. Shanahan, Nature 379, 432 (1996)

158. S. Schiaffino, A.A. Sonin, Phys. Fluids 9, 2217 (1997)

159. F. Tavakoli, S.H. Davis, H.P. Kavehpour, Langmuir 30, 10151 (2014)

160. R. de Ruiter, P. Colinet, P. Brunet, J.H. Snoeijer, H. Gelderblom, Phys. Rev. Fluids 2, $043602(2017)$

161. R. Herbaut, P. Brunet, L. Limat, L. Royon, Phys. Rev. Fluids 4, 033603 (2019) 033603.

162. S. Schiaffino, A.A. Sonin, Phys. Fluids 9, 2227 (1997)

163. V. Thiévenaz, T. Séon, C. Josserand, J. Fluid Mech. 874, 756 (2019)

164. F.G. Yost, P.A. Sackenger, E.J. O'Toole, Acta Mater. 46, 2329 (1998)

165. J.A. Warren, W.J. Boettinger, A. Roosen, Acta Mater. 46, 3247 (1998)

166. W. Villanueva, K. Grönbagen, G. Amberg, J. Agren, Phys. Rev. E 77, 056313 (2008)

167. W. Villanueva, W.J. Boettinger, J.A. Warren, G. Amberg, Acta Mater. 57, 6022 (2009)

168. T.J. Singler, S. Su, L. Yin, B.T. Murray, J. Mater. Sci. 47, 8261 (2012)

169. A. Rednikov, P. Colinet, Phys. Rev. E 87, 010401 (2013)

170. V. Janeček, F. Doumenc, B. Gourrier, V.S. Nikolayev, J. Colloid Interface Sci. 460, $329(2015)$

171. X. Xu, T. Qian, Phys. Rev. E 85, 061603 (2012)

172. P.-G. de Gennes, C. R. Acad. Sci. Paris 297, 9 (1983)

173. H. Hervet, P.-G. de Gennes, C. R. Acad. Sci. Paris 299, 499 (1984)

174. T.D. Blake, Y.D. Shikhmurzaev, J. Colloid Interface Sci. 253, 196 (2002) 